\title{
A pathwise approach to the extinction of branching processes with countably many types
}

\author{
Peter Braunsteins $^{\mathrm{a}}$, Geoffrey Decrouez ${ }^{\mathrm{b}}$, Sophie Hautphenne ${ }^{\mathrm{a}, \mathrm{c}, *}$ \\ ${ }^{a}$ The University of Melbourne, Australia \\ ${ }^{b}$ National Research University, Higher School of Economics, Russia \\ ${ }^{c}$ Swiss Federal Institute of Technology Lausanne, Switzerland
}

\begin{abstract}
We consider the extinction events of Galton-Watson processes with countably infinitely many types. In particular, we construct truncated and augmented Galton-Watson processes with finite but increasing sets of types. A pathwise approach is then used to show that, under some sufficient conditions, the corresponding sequence of extinction probability vectors converges to the global extinction probability vector of the Galton-Watson process with countably infinitely many types. Besides giving rise to a family of new iterative methods for computing the global extinction probability vector, our approach paves the way to new global extinction criteria for branching processes with countably infinitely many types.
\end{abstract}

Keywords: multitype branching process, extinction probability, pathwise approach, extinction criterion 2010 MSC: 60J80, 60J05, 60J22, 65H10

\section{Introduction}

Multitype Galton-Watson branching processes (MGWBPs) are stochastic models describing the evolution of a population of individuals who live one

\footnotetext{
*Corresponding author

Email addresses: p.braunsteins@student.unimelb.edu.au (Peter Braunsteins), ggdecrouez@hse.ru (Geoffrey Decrouez), sophiemh@unimelb.edu.au (Sophie Hautphenne)
} 
unit of time and give birth to a random number of offspring that may be of various types. Each type may have a different progeny distribution, and individuals behave independently of each other. These processes have been studied extensively during the last decades; classical reference books include Harris [15], Mode [23], Athreya and Ney [1], and Jagers [18]. MGWBPs have numerous applications and have been used to model important problems arising in biology, ecology, physics and even computer science. Recent books with special emphasis on applications are Axelrod and Kimmel [3], and Haccou, Jagers and Vatutin [14].

One of the main topics of research on MGWBPs, and on branching processes in general, is the characterisation of the probability that the population eventually becomes empty. We denote by $q_{i}$ the conditional extinction probability of the branching process, given that it starts with a single individual of type $i$, and we let $\boldsymbol{q}:=\left(q_{i}\right)$. When the number of types is finite, it is well known that the vector $\boldsymbol{q}$ is the minimal non-negative solution of the fixed-point extinction equation,

$$
s=G(s),
$$

where $\boldsymbol{G}(\boldsymbol{s}):=\left(G_{i}(\boldsymbol{s})\right)$ records the progeny generating function associated with each type. Most of the time this finite system of equations cannot be solved analytically, but the linear functional iteration algorithm or the quadratic Newton algorithm can be applied to compute $\boldsymbol{q}$ numerically. In addition, there is a well-established extinction criterion, namely $\boldsymbol{q}=\mathbf{1}$ if and only if the Perron-Frobenius eigenvalue of the mean progeny matrix is less than or equal to one.

In contrast to the finite-type case, when there are infinitely many types we need to distinguish between different extinction events. We say that there is global extinction when the whole population becomes extinct, and $\boldsymbol{q}$ corresponds to the probability vector for this event. We refer to the event that every type becomes extinct as partial extinction, and denote its probability vector by $\tilde{\boldsymbol{q}}$. In the context of branching random walks (BRWs), in which individuals are assigned locations instead of types, partial extinction is analogous to local extinction at every location. It is clear that global extinction implies partial extinction but the converse does not necessarily hold. Indeed, it is possible for every type to eventually disappear while the total number of individuals approaches infinity, see for instance [16, Section 5]. The vectors $\boldsymbol{q}$ and $\tilde{\boldsymbol{q}}$ both satisfy (1.1), where $\boldsymbol{q}$ is the minimal non-negative solution and 
$\tilde{\boldsymbol{q}}$ may or may not be equal to $\boldsymbol{q}$.

Due to the challenges that arise when transitioning from a finite to a countably infinite type set, many of the questions that have been thoroughly explored in the finite setting remain open. Indeed, apart from the recent works of [21] and [28] on a restricted class of MGWBPs with linear fractional progeny generating functions, and of [16] on algorithmic techniques, scant attention has been paid to computational aspects of the infinite extinction probability vectors $\boldsymbol{q}$ and $\tilde{\boldsymbol{q}}$. In addition, several authors have investigated conditions for $\boldsymbol{q}=\mathbf{1}$ or $\tilde{\boldsymbol{q}}=\mathbf{1}$, see for instance [6, 15, 16, 25, 26, 30, 32, 35]. However, while it has been well established that the convergence norm of the infinite mean progeny matrix provides a partial extinction criterion, see [27], the literature still lacks an easily applicable global extinction criterion that holds under mild conditions. Here we address the two problems in parallel by defining two new probabilistic tools: to each MGWBP with countably many types $\left\{\boldsymbol{Z}_{n}\right\}$, we associate (i) a sequence of truncated and augmented finitetype branching processes $\left\{\overline{\boldsymbol{Z}}_{n}^{(k)}\right\}_{k \geq 1, n \geq 0}$, which themselves naturally define (ii) an embedded branching process $\left\{\boldsymbol{S}_{k}\right\}$ referred to as the seed process. The next two paragraphs provide an intuitive description of these two processes and their benefits.

For each $k \geq 1$, the $k$ th finite-type branching process $\left\{\overline{\boldsymbol{Z}}_{n}^{(k)}\right\}$ is constructed pathwise on the same probability space as the original process $\left\{\boldsymbol{Z}_{n}\right\}$, by replacing all types larger than $k$ with a type randomly selected from the set $\{1, \ldots, k\}$ according to some distribution $\boldsymbol{\alpha}^{(k)}$. The corresponding finite extinction probability vector is denoted by $\overline{\boldsymbol{q}}^{(k)}$. In our main theorem (Theorem 1 ), we prove that, under some sufficient conditions on $\left\{\boldsymbol{Z}_{n}\right\}$ (closely related to the dichotomy between extinction and unbounded growth of the population), and on the sequence of replacement distributions $\left\{\boldsymbol{\alpha}^{(k)}\right\}_{k \geq 1}$ (similar to a tightness condition), the sequence $\left\{\overline{\boldsymbol{q}}^{(k)}\right\}_{k \geq 1}$ converges to the global extinction probability $\boldsymbol{q}$. This result establishes a link between the extinction of non-singular irreducible finite-type branching processes and global extinction in the infinite-type setting. It has several implications. First, Theorem 1 extends the work in [16], in which two monotone sequences of extinction probability vectors, $\left\{\boldsymbol{q}^{(k)}\right\}_{k \geq 1}$ and $\left\{\tilde{\boldsymbol{q}}^{(k)}\right\}_{k \geq 1}$, are shown to converge respectively to $\boldsymbol{q}$ and $\tilde{\boldsymbol{q}}$. These sequences were obtained by replacing all types larger than $k$ either by an immortal type (yielding $\boldsymbol{q}^{(k)}$ ), or by a sterile type (yielding $\tilde{\boldsymbol{q}}^{(k)}$ ), and the monotone convergence theorem was the main argument in the proof. In contrast, the new sequence $\left\{\overline{\boldsymbol{q}}^{(k)}\right\}$ is not necessarily monotone, 
and a completely different approach is required. From a computational point of view, the flexibility in the choice of the replacement distributions $\left\{\boldsymbol{\alpha}^{(k)}\right\}$ motivates the search for an optimal choice maximising the convergence rate, but this is out of the scope of this paper. Second, as a direct consequence of Theorem 1, we derive new sufficient conditions for $\boldsymbol{q}=\mathbf{1}$ and $\boldsymbol{q}<\mathbf{1}$. Such results could not be obtained using the sequences in [16].

The seed process $\left\{\boldsymbol{S}_{k}\right\}$ is an MGWBP evolving in a varying environment, that arises naturally when exploring the asymptotic behaviour of $\left\{\overline{\boldsymbol{q}}^{(k)}\right\}$. It is constructed pathwise from the family of finite-type processes $\left\{\overline{\boldsymbol{Z}}_{n}^{(k)}\right\}$ as follows: the individuals (or seeds) in the $k$ th generation of $\left\{\boldsymbol{S}_{k}\right\}$ correspond to the individuals in $\left\{\boldsymbol{Z}_{n}\right\}$ which are replaced by a random type according to $\boldsymbol{\alpha}^{(k)}$ to form $\left\{\overline{\boldsymbol{Z}}_{n}^{(k)}\right\}$. The seed process is the fundamental ingredient in the proof of Theorem 1, but in addition it enjoys several interesting properties on its own. For example, $\left\{\boldsymbol{S}_{k}\right\}$ almost surely becomes extinct if and only if the global and partial extinction probability vectors of the original process coincide. While in the present paper our interest in the seed process remains its application to the sequence $\left\{\overline{\boldsymbol{q}}^{(k)}\right\}$, we lay the foundations for a subsequent paper [8], in which properties of the seed process are exploited further, to yield, among other results, a global extinction criterion that applies to a class of branching processes referred to as lower Hessenberg.

Finally, we investigate the asymptotic properties of the sequence $\left\{\overline{\boldsymbol{q}}^{(k)}\right\}$ when the conditions on $\left\{\boldsymbol{\alpha}^{(k)}\right\}$ in Theorem 1 are not met. We consider (a) replacement by the last type, that is, $\boldsymbol{\alpha}^{(k)}=\boldsymbol{e}_{k}$, and (b) replacement by a uniformly distributed type, $\boldsymbol{\alpha}^{(k)}=1 / k$, and one particular example that focuses on each case, Examples 2 and 3, respectively. In Example 2, we prove that the limit of the sequence $\left\{\overline{\boldsymbol{q}}^{(k)}\right\}$ does not always exist, and in Example 3, the limit does exist but may correspond to the partial extinction probability $\tilde{\boldsymbol{q}}$. Example 2 highlights the sensitivity of the limit of $\left\{\overline{\boldsymbol{q}}^{(k)}\right\}$ under (a), whereas Example 3 demonstrates how alternative choices of $\left\{\boldsymbol{\alpha}^{(k)}\right\}$ may lead to contrasting asymptotic behaviour in $\left\{\overline{\boldsymbol{q}}^{(k)}\right\}$.

The paper is organised as follows. In Section 2 we provide background on MGWBPs with countably many types. In Section 3 we focus on the pathwise construction of the branching processes with corresponding extinction probabilities $\boldsymbol{q}^{(k)}, \tilde{\boldsymbol{q}}^{(k)}$, and $\overline{\boldsymbol{q}}^{(k)}$. In Section 4 we establish sufficient conditions for the convergence of $\left\{\overline{\boldsymbol{q}}^{(k)}\right\}$ to $\boldsymbol{q}$, we study properties of the related seed process, and we prove the main theorem on the convergence of $\left\{\overline{\boldsymbol{q}}^{(k)}\right\}$. 
In Section 5 we derive sufficient conditions for $\boldsymbol{q}=\mathbf{1}$ and $\boldsymbol{q}<\mathbf{1}$. Finally, in Section 6 we study the asymptotic behaviour of $\left\{\overline{\boldsymbol{q}}^{(k)}\right\}$ for replacement distributions that do not satisfy the conditions of our main theorem and provide some numerical illustrations. The pseudo-code for the computation of the global and partial extinction probabilities, and the proofs related to Examples 2 and 3 are provided in some appendices.

\section{Preliminaries}

Consider a multitype Galton-Watson process with countably infinite type set $\mathcal{S}=\{1,2,3, \ldots\}$. Throughout the paper we assume that the process initially contains a single individual, whose type is denoted by $\varphi_{0}$. The process then evolves according to the following rules:

(i) each individual lives for a single generation, and

(ii) at death it gives birth to $\boldsymbol{r}=\left(r_{1}, r_{2}, \ldots\right)$ offspring, that is, $r_{1}$ individuals of type $1, r_{2}$ individuals of type 2 , etc., where the vector $\boldsymbol{r}$ is chosen independently of all other individuals according to a probability distribution, $p_{i}(\cdot)$, specific to the parental type $i \in \mathcal{S}$.

Following Mode [23] we now give an equivalent but more formal construction of this process. This formulation differs slightly from the standard construction in Harris [15] and Jagers [19] but is useful, in particular, in defining the sequence of truncated and augmented processes in Section 3.

Consider the set of all possible individuals of the form $\left\langle i_{1} j_{1}\right\rangle,\left\langle i_{1} j_{1} i_{2} j_{2}\right\rangle$, $\ldots,\left\langle i_{1} j_{1} i_{2} j_{2} \ldots i_{n} j_{n}\right\rangle, \ldots$ where $\left\langle i_{1} j_{1} i_{2} j_{2} \ldots i_{n} j_{n}\right\rangle$ is a member of the $n$th generation and is the $i_{n}$ th child of type $j_{n}$ born to $\left\langle i_{1} j_{1} i_{2} j_{2} \ldots i_{n-1} j_{n-1}\right\rangle$. In other words, each individual in the $n$th generation belongs to the set $\mathcal{J}_{n}=$ $\langle\mathbb{N} \times \mathcal{S}\rangle^{n}$, and $\mathcal{J}=\langle 0\rangle \cup \bigcup_{n=1}^{\infty} \mathcal{J}_{n}$ contains all individuals. To each individual $I \in \mathcal{J}$ we associate a sample space $\Omega_{I}$ made up of all infinite, non-negative, integer-valued vectors $\boldsymbol{r}_{I}$, with at most finitely many strictly positive entries, where $\boldsymbol{r}_{I}$ represents the number of offspring of the various types produced by the individual. Let $\mathcal{B}_{I}$ be the corresponding discrete $\sigma$-algebra, and let $\mathbf{P}_{I}$ be the probability measure such that $\mathbf{P}_{I}(B)=\sum_{\boldsymbol{r}_{I} \in B} p_{i}\left(\boldsymbol{r}_{I}\right)$ if $I$ is of type $i$, for all $B \in \mathcal{B}_{I}$. The sequence of probability spaces $\left\{\left(\Omega_{I}, \mathcal{B}_{I}, \mathbf{P}_{I}\right)\right\}_{I \in \mathcal{J}}$ induces the product probability space $(\Omega, \mathcal{F}, \mathbf{P})$ on which the Galton-Watson branching 
process with countably many types is defined. The elements $\omega \in \Omega$ are of the form $\omega=\left(\boldsymbol{r}_{I} ; I \in \mathcal{J}\right)$. Let $\boldsymbol{N}(\omega, I)=\left(N_{1}(\omega, I), N_{2}(\omega, I), \ldots\right)$ contain the number of offspring of each type generated by individual $I$. The individual $I=\left\langle i_{1} j_{1} \ldots i_{n} j_{n}\right\rangle$ then appears in the population if and only if,

$$
i_{1} \leq N_{j_{1}}(\omega,\langle 0\rangle), \quad i_{2} \leq N_{j_{2}}\left(\omega,\left\langle i_{1} j_{1}\right\rangle\right), \ldots, \quad i_{n} \leq N_{j_{n}}\left(\omega,\left\langle i_{1} j_{1} \ldots i_{n-1} j_{n-1}\right\rangle\right)
$$

For $I=\left\langle i_{1} j_{1} \ldots i_{n} j_{n}\right\rangle \in \mathcal{J}_{n}$, let $Z_{j}(\omega, I)=1$ if both $j_{n}=j$ and condition (2.1) are satisfied, and equal 0 otherwise. The population at generation $n$ is then given by the vector $\boldsymbol{Z}_{n}(\omega)$ which has entries

$$
Z_{n, j}(\omega)=\sum_{I \in \mathcal{J}_{n}} Z_{j}(\omega, I), \quad j \in \mathcal{S}
$$

In the sequel, we will often drop the dependence in $\omega$ when it is not contextually important, and refer to the branching process as $\left\{\boldsymbol{Z}_{n}\right\}_{n \geq 0}$. We let $\left|\boldsymbol{Z}_{n}\right|:=\sum_{j \in \mathcal{S}} Z_{n, j}$ be the total population size at generation $n$.

From the set of probability distributions $\left\{p_{i}(\cdot)\right\}_{i \in \mathcal{S}}$, we define the progeny generating function $\boldsymbol{G}:[0,1]^{\mathcal{S}} \rightarrow[0,1]^{\mathcal{S}}$, which has entries,

$$
G_{i}(\boldsymbol{s})=\sum_{\boldsymbol{r}} p_{i}(\boldsymbol{r}) \boldsymbol{s}^{\boldsymbol{r}}=\sum_{\boldsymbol{r}} p_{i}(\boldsymbol{r}) \prod_{k=1}^{\infty} s_{k}^{r_{k}}, \quad i \in \mathcal{S} .
$$

The mean progeny matrix $M$ is an infinite matrix whose entries are given by

$$
M_{i j}=\left.\frac{\partial G_{i}(s)}{\partial s_{j}}\right|_{s=\mathbf{1}}, \quad \text { for } i, j \in \mathcal{S},
$$

where $M_{i j}$ corresponds to the expected number of type- $j$ children born to a parent of type $i$. We assume that the row sums of $M$ are finite, that is, the expected total number of direct offspring of an individual of any type is finite. It is sometimes convenient to associate a graph to the mean progeny matrix whose set of vertices corresponds to the set of types $\mathcal{S}$, and in which there is an oriented edge between nodes $i$ and $j$ with weight $M_{i j}$ if and only if $M_{i j}>0$. We shall refer later to this graph as the mean progeny representation graph. We say that there is a path from type $i$ to type $j$ if such a directed path exists in the mean progeny representation graph. The process $\left\{\boldsymbol{Z}_{n}\right\}$ is irreducible if there is a path between every pair of nodes. In the context of countably infinitely many types, properties of the non-negative matrix $M$ have been 
studied mainly in the $R$-recurrent case, see for instance $[24,33,34]$. In this paper we do not make any assumption on $R$-recurrence or $R$-transience, our results hold in both cases.

We distinguish between two types of extinction events: the global extinction event, $\left\{\lim _{n \rightarrow \infty}\left|\boldsymbol{Z}_{n}\right|=0\right\}$, corresponding to the event that the whole population eventually becomes extinct, and the partial extinction event, $\left\{\forall l \geq 1: \lim _{n \rightarrow \infty} Z_{n, l}=0\right\}$, corresponding to the event that all types eventually become extinct. Note that in the finite-type case, both events are equivalent. The conditional global extinction probability vector, given the initial type, is $\boldsymbol{q}=\left(q_{1}, q_{2}, \ldots\right)$, where

$$
q_{i}=\mathbf{P}\left(\lim _{n \rightarrow \infty}\left|\boldsymbol{Z}_{n}\right|=0 \mid \varphi_{0}=i\right),
$$

and the conditional partial extinction probability vector, given the initial type, is $\tilde{\boldsymbol{q}}=\left(\tilde{q}_{1}, \tilde{q}_{2}, \ldots\right)$, where

$$
\tilde{q}_{i}=\mathbf{P}\left(\forall l \geq 1: \lim _{n \rightarrow \infty} Z_{n, l}=0 \mid \varphi_{0}=i\right) .
$$

It is clear that global extinction implies partial extinction, therefore $\boldsymbol{q} \leq \tilde{\boldsymbol{q}}$. The vectors $\boldsymbol{q}$ and $\tilde{\boldsymbol{q}}$ are both solutions to the fixed point equation (1.1). Additionally, $\boldsymbol{q}$ is the minimal non-negative solution of (1.1), whereas $\tilde{\boldsymbol{q}}$ is not necessarily the minimal non-negative solution.

In our illustrative examples we shall make use of a process defined for any MGWBP $\boldsymbol{V}:=\left\{\boldsymbol{V}_{n}\right\}_{n \geq 0}$ with type set $\mathcal{S}_{V} \subseteq \mathcal{S}$, which was previously considered by $[4,9,10]$, among others. We refer to this process as the type-i branching process embedded with respect to $\left\{\boldsymbol{V}_{n}\right\}_{n \geq 0}$ and denote it by $\left\{E_{n}^{(i)}(\boldsymbol{V})\right\}_{n \geq 0}$. The sample paths of $\left\{E_{n}^{(i)}(\boldsymbol{V})\right\}$ are constructed from those of $\left\{\boldsymbol{V}_{n}: \varphi_{0}=i\right\}$ by taking all type- $i$ individuals that appear in $\left\{\boldsymbol{V}_{n}\right\}$ and defining the direct descendants of these individuals as their closest (in generation) type- $i$ descendants in $\left\{\boldsymbol{V}_{n}\right\}$. The process $\left\{E_{n}^{(i)}(\boldsymbol{V})\right\}$ evolves as a single-type GaltonWatson process whose extinction probability is equivalent to the probability that type $i$ becomes extinct in $\left\{\boldsymbol{V}_{n}\right\}$. While we will use this fact directly in Example 1, it also implies that type $i$ survives with positive probability in $\left\{\boldsymbol{V}_{n}\right\}$ if and only if the mean number of offspring in $\left\{E_{n}^{(i)}(\boldsymbol{V})\right\}$ is strictly greater than 1 , that is,

$$
m_{E_{n}^{(i)}(\boldsymbol{V})}:=M_{i i}+\sum_{n=2}^{\infty}\left(\sum_{i_{1}, \ldots, i_{n-1} \in \mathcal{S} \backslash\{i\}} M_{i i_{1}} M_{i_{1} i_{2}} \ldots M_{i_{n-1} i}\right)>1 .
$$


Observe that $m_{E_{n}^{(i)}(\boldsymbol{V})}$ can be identified as the weighted sum of all first return paths to $i$ in the mean progeny representation graph associated with the branching process $\left\{\boldsymbol{V}_{n}\right\}$. In the irreducible case, if $\left|\mathcal{S}_{V}\right|<\infty$, then

$$
m_{E_{n}^{(i)}(\boldsymbol{V})} \leq 1 \quad \Leftrightarrow \quad \rho(M) \leq 1 \quad \Leftrightarrow \quad \boldsymbol{q}=\tilde{\boldsymbol{q}}=\mathbf{1},
$$

where $\rho(M)$ denotes the Perron-Frobenius eigenvalue of the mean progeny matrix $M$. If $\left|\mathcal{S}_{V}\right|=\infty$, then

$$
m_{E_{n}^{(i)}(\boldsymbol{V})} \leq 1 \quad \Leftrightarrow \quad \nu(M) \leq 1 \quad \Leftrightarrow \quad \tilde{\boldsymbol{q}}=\mathbf{1},
$$

where $\nu(M)$ denotes the convergence norm of $M$; see for instance [35].

In the sequel, we adopt the shorthand notation $\mathbf{P}_{i}(\cdot):=\mathbf{P}\left(\cdot \mid \varphi_{0}=i\right)$ and $\mathbf{E}_{i}(\cdot):=\mathbf{E}\left(\cdot \mid \varphi_{0}=i\right)$. For any $k \geq 1$, we define the partition $T_{k}:=\{1,2, \ldots, k\}$ and $T_{k}^{c}:=\{k+1, k+2, \ldots\}$ of the set of types $\mathcal{S}=\{1,2,3, \ldots\}$, and we let

$$
\tau_{k}(\omega)=\inf \left\{n \geq 0: \sum_{i=k+1}^{\infty} Z_{n, i}(\omega)>0\right\}
$$

be the first passage time to $T_{k}^{c}$. Note that for each $\omega \in \Omega,\left\{\tau_{k}(\omega)\right\}_{k \geq 1}$ forms a increasing sequence in $k$. Finally we denote by $\boldsymbol{e}_{i}$ the vector with $i$-th entry 1 and all other entries 0 , and whose length will be defined by the context.

\section{Pathwise construction of finite-type branching processes on $(\Omega, \mathcal{F}, \mathrm{P})$}

In this section we construct the sequences of finite-type branching processes $\left\{\tilde{\boldsymbol{Z}}_{n}^{(k)}\right\}_{n \geq 0},\left\{\boldsymbol{Z}_{n}^{(k)}\right\}_{n \geq 0}$ and $\left\{\overline{\boldsymbol{Z}}_{n}^{(k)}\right\}_{n \geq 0}$ on $(\Omega, \mathcal{F}, \mathbf{P})$. The first two sequences were studied in [16], while the latter has not been previously considered. This construction, which was not detailed in [16], plays a key role in the remainder of the paper.

For each $k \geq 1$, the realisations of $\left\{\tilde{\boldsymbol{Z}}_{n}^{(k)}\right\}$ are constructed pathwise from those of $\left\{\boldsymbol{Z}_{n}\right\}$ by removing the descendants of all individuals of type $i \in T_{k}^{c}$. More specifically, for each $\omega \in \Omega$ and individual $I=\left\langle i_{1} j_{1} \ldots i_{n} j_{n}\right\rangle \in \mathcal{J}$ of type $j=j_{n}$, we let $\tilde{\boldsymbol{N}}^{(k)}(\omega, I)=\boldsymbol{N}(\omega, I) \mathbb{1}\{j \leq k\}$. The condition of appearance of an individual in the truncated branching process is then the same as (2.1), replacing $N_{j} .(\omega, I)$ by $\tilde{N}_{j}^{(k)}(\omega, I)$, and the definition of the population size vector $\tilde{\boldsymbol{Z}}_{n}^{(k)}(\omega)$ is analogous to $(2.2)$. Consequently, in $\left\{\tilde{\boldsymbol{Z}}_{n}^{(k)}\right\}$ 
(i) all types in $T_{k}$ have the same progeny as the corresponding types in $\left\{\boldsymbol{Z}_{n}\right\}$, and

(ii) all types in $T_{k}^{c}$ die with no offspring; these types are said to be sterile.

We denote by $\tilde{\boldsymbol{q}}^{(k)}$ the global extinction probability vector of $\left\{\tilde{\boldsymbol{Z}}_{n}^{(k)}\right\}$. Since all types larger than $k$ are sterile in $\left\{\tilde{\boldsymbol{Z}}_{n}^{(k)}\right\}$, the truncated process behaves effectively like a finite-type branching process on the set of types $\{1,2, \ldots, k\}$. It is clear that $\tilde{q}_{i}^{(k)}=1$ for all $i>k$, so the computation of $\tilde{\boldsymbol{q}}^{(k)}$ reduces to solving a finite system of $k$ equations. It was shown in [16] that the sequence $\left\{\tilde{\boldsymbol{q}}^{(k)}\right\}$ is monotone decreasing and converges pointwise to $\tilde{\boldsymbol{q}}$.

Similarly, for each $k \geq 1$ the process $\left\{\boldsymbol{Z}_{n}^{(k)}\right\}$ is constructed pathwise from realisations of $\left\{\boldsymbol{Z}_{n}\right\}$ by removing all individuals of type $i \in T_{k}^{c}$ and their descendants, and replacing each pruned branch with an infinite line of descent made up of type- $\Delta$ individuals. More formally, for each $\omega \in \Omega$, the population size vector $\boldsymbol{Z}_{n}^{(k)}(\omega)$ has entries

$$
Z_{n, i}^{(k)}(\omega)= \begin{cases}\sum_{j=0}^{n} \sum_{l=k+1}^{\infty} \tilde{Z}_{j, l}^{(k)}(\omega), & i=\Delta \\ \tilde{Z}_{n, i}^{(k)}(\omega), & 1 \leq i \leq k \\ 0, & i>k\end{cases}
$$

As a consequence, in $\left\{\boldsymbol{Z}_{n}^{(k)}\right\}$,

(i) all types in $T_{k}$ have the same progeny distribution as the corresponding types in $\left\{\boldsymbol{Z}_{n}\right\}$, and

(ii) all types in $T_{k}^{c}$ are instantaneously replaced by the absorbing type $\Delta$, which at each generation produces a single type- $\Delta$ progeny with probability one.

Once a type- $\Delta$ individual is born, $\left\{\boldsymbol{Z}_{n}^{(k)}\right\}$ does not become extinct. In this sense, individuals of type $\Delta$ can be thought of as immortal. We denote by $\boldsymbol{q}^{(k)}$ the global extinction probability vector of $\left\{\boldsymbol{Z}_{n}^{(k)}\right\}$. It contains only finitely many non-zero entries since $q_{i}^{(k)}=0$ for all $i>k$. It is clear that $\left\{\boldsymbol{Z}_{n}^{(k)}\right\}$ becomes extinct if and only if $\left\{\boldsymbol{Z}_{n}\right\}$ becomes extinct before the birth of the first individual with a type in $T_{k}^{c}$. It was proved in [16] that the sequence $\left\{\boldsymbol{q}^{(k)}\right\}$ is monotone increasing and converges pointwise to $\boldsymbol{q}$. 
For each $k \geq 1$, we construct recursively the truncated and augmented branching process $\left\{\overline{\boldsymbol{Z}}_{n}^{(k)}\right\}_{n \geq 0}$ for which

(i) all types in $T_{k}$ have the same progeny distribution as the corresponding types in $\left\{\boldsymbol{Z}_{n}\right\}$, and

(ii) all types in $T_{k}^{c}$ are instantaneously, and independently of each other, replaced by type $X \in\{1, \ldots, k\}$ which is selected using the probability distribution $\boldsymbol{\alpha}^{(k)}$. The replaced individual then generates new individuals according to the progeny distribution of its type.

To construct the sample paths of $\left\{\overline{\boldsymbol{Z}}_{n}^{(k)}\right\}_{n \geq 0}$ we first augment the probability space $(\Omega, \mathcal{F}, \mathbf{P})$ to carry the sequence of independent random vari-

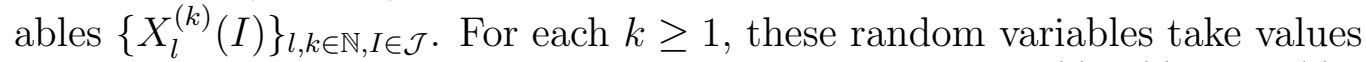
in $\{1, \ldots, k\}$ and have probability distribution $\boldsymbol{\alpha}^{(k)}=\left(\alpha_{1}^{(k)}, \alpha_{2}^{(k)}, \ldots, \alpha_{k}^{(k)}\right)$, where $\alpha_{i}^{(k)}=\mathbf{P}\left(X_{l}^{(k)}(I)=i\right)$. We interpret $X_{l}^{(k)}(\omega, I)$ as the replacement type of the $l$ th offspring of type strictly larger than $k$ born to $I$ for the realisation $\omega$. Let $N_{(k, \infty)}(\omega, I)=\sum_{j=k+1}^{\infty} N_{j}(\omega, I)$ and define $\overline{\boldsymbol{N}}^{(k)}(\omega, I)$ with entries,

$$
\bar{N}_{i}^{(k)}(\omega, I)= \begin{cases}N_{i}(\omega, I)+\sum_{l=1}^{N_{(k, \infty)}(\omega, I)} 1\left\{X_{l}^{(k)}(\omega, I)=i\right\}, & 1 \leq i \leq k \\ 0, & i>k .\end{cases}
$$

The condition of appearance of an individual in the truncated and augmented branching process is then the same as $(2.1)$, replacing $N_{j} .(\omega, I)$ by $\bar{N}_{j}^{(k)}(\omega, I)$, and the definition of the population size vector $\overline{\boldsymbol{Z}}_{n}^{(k)}(\omega)$ is analogous to (2.2). An illustration of $\left\{\boldsymbol{Z}_{n}\right\},\left\{\tilde{\boldsymbol{Z}}_{n}^{(k)}\right\},\left\{\boldsymbol{Z}_{n}^{(k)}\right\}$ and $\left\{\overline{\boldsymbol{Z}}_{n}^{(k)}\right\}$ is given in Figure 3 for $k=2$ and a specific $\omega \in \Omega$.

We denote by $\overline{\boldsymbol{q}}^{(k)}$ the global extinction probability vector of $\left\{\overline{\boldsymbol{Z}}_{n}^{(k)}\right\}$. The vector $\overline{\boldsymbol{q}}^{(k)}$ contains infinitely many entries, and is such that for all $i>k, \bar{q}_{i}^{(k)}=\sum_{j=1}^{k} \alpha_{j}^{(k)} \bar{q}_{j}^{(k)}$; this represents the probability that the daughter process of a replaced individual becomes extinct, and will be denoted by $\boldsymbol{\alpha}^{(k)} \overline{\boldsymbol{q}}^{(k)}:=\sum_{j=1}^{k} \alpha_{j}^{(k)} \bar{q}_{j}^{(k)}$ in the sequel. The computation of $\overline{\boldsymbol{q}}^{(k)}$ reduces to finding the extinction probability vector of a MGWBP with a finite type set. The pseudo-code for the computation of the three sequences $\left\{\boldsymbol{q}^{(k)}\right\},\left\{\tilde{\boldsymbol{q}}^{(k)}\right\}$, and $\left\{\overline{\boldsymbol{q}}^{(k)}\right\}$ is provided in Appendix A.

The goal of the next section is to determine sufficient conditions for the convergence of the sequence $\left\{\overline{\boldsymbol{q}}^{(k)}\right\}_{k \geq 1}$ to $\boldsymbol{q}$. Unlike the sequences $\left\{\boldsymbol{q}^{(k)}\right\}$ and 


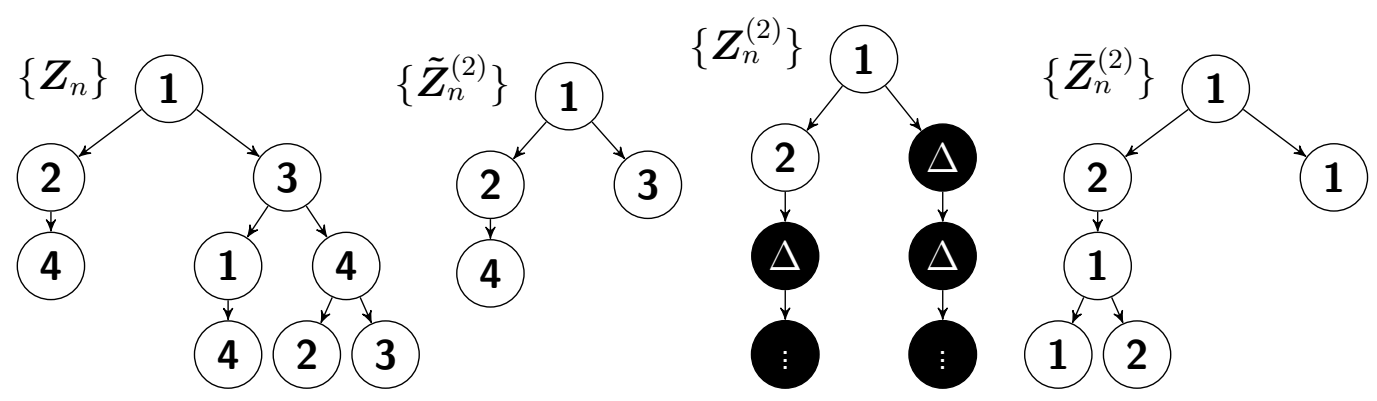

Figure 3.1: A visualisation of $\left\{\boldsymbol{Z}_{n}\right\},\left\{\tilde{\boldsymbol{Z}}_{n}^{(2)}\right\},\left\{\boldsymbol{Z}_{n}^{(2)}\right\}$ and $\left\{\overline{\boldsymbol{Z}}_{n}^{(2)}\right\}$ corresponding to a specific realisation $\omega \in \Omega$. It is such that $\boldsymbol{r}_{\langle 0\rangle}=\boldsymbol{e}_{2}+\boldsymbol{e}_{3}, \boldsymbol{r}_{\langle 12\rangle}=\boldsymbol{e}_{4}, \boldsymbol{r}_{\langle 13\rangle}=\boldsymbol{e}_{1}+\boldsymbol{e}_{4}, \boldsymbol{r}_{\langle 1311\rangle}=\boldsymbol{e}_{4}$, $\boldsymbol{r}_{\langle 1314\rangle}=\boldsymbol{e}_{2}+\boldsymbol{e}_{3}$ and $\boldsymbol{r}_{\langle 1214\rangle}=\boldsymbol{r}_{\langle 131114\rangle}=\boldsymbol{r}_{\langle 131412\rangle}=\boldsymbol{r}_{\langle 131413\rangle}=\mathbf{0}$ are used to construct $\left\{\boldsymbol{Z}_{n}\right\},\left\{\tilde{\boldsymbol{Z}}_{n}^{(2)}\right\}$ and $\left\{\boldsymbol{Z}_{n}^{(2)}\right\}$, and additionally $X_{1}^{(2)}(\omega,\langle 0\rangle)=1, \boldsymbol{r}_{\langle 11\rangle}=\mathbf{0}, X_{1}^{(2)}(\omega,\langle 12\rangle)=1$, $\boldsymbol{r}_{\langle 1211\rangle}=\boldsymbol{e}_{1}+\boldsymbol{e}_{2}$ and $\boldsymbol{r}_{\langle 121111\rangle}=\boldsymbol{r}_{\langle 121112\rangle}=\mathbf{0}$ are used to construct $\left\{\overline{\boldsymbol{Z}}_{n}^{(k)}\right\}$. All other information contained in $\omega$ is not required.

$\left\{\tilde{\boldsymbol{q}}^{(k)}\right\}$, the convergence of the sequence $\left\{\overline{\boldsymbol{q}}^{(k)}\right\}$ may not be monotone. We show in the next lemma that $\left\{\overline{\boldsymbol{q}}^{(k)}\right\}$ is however always caught between $\left\{\boldsymbol{q}^{(k)}\right\}$ and $\left\{\tilde{\boldsymbol{q}}^{(k)}\right\}$.

Lemma 1. For any $k \geq 1$ and replacement distribution $\boldsymbol{\alpha}^{(k)}$,

$$
\boldsymbol{q}^{(k)} \leq \overline{\boldsymbol{q}}^{(k)} \leq \tilde{\boldsymbol{q}}^{(k)}
$$

Proof. From the pathwise construction of the branching processes, it is clear that

$$
\left\{\omega: \lim _{n \rightarrow \infty}\left|\boldsymbol{Z}_{n}^{(k)}(\omega)\right|=0\right\} \subseteq\left\{\omega: \lim _{n \rightarrow \infty}\left|\overline{\boldsymbol{Z}}_{n}^{(k)}(\omega)\right|=0\right\} \subseteq\left\{\omega: \lim _{n \rightarrow \infty}\left|\tilde{\boldsymbol{Z}}_{n}^{(k)}(\omega)\right|=0\right\},
$$

and the result follows.

Corollary 1. For any sequence $\left\{\boldsymbol{\alpha}^{(k)}\right\}$ of replacement distributions,

$$
\boldsymbol{q} \leq \liminf _{k \rightarrow \infty} \overline{\boldsymbol{q}}^{(k)} \leq \limsup _{k \rightarrow \infty} \overline{\boldsymbol{q}}^{(k)} \leq \tilde{\boldsymbol{q}}
$$

Proof. The result is immediate by Lemma 1 since $\boldsymbol{q}^{(k)} \rightarrow \boldsymbol{q}$ and $\tilde{\boldsymbol{q}}^{(k)} \rightarrow \tilde{\boldsymbol{q}}$.

A consequence of Corollary 1 is that, when it exists, the limit of the sequence $\left\{\overline{\boldsymbol{q}}^{(k)}\right\}$ can only overestimate the probability of global extinction. In Section 4.1, we illustrate a situation where $\boldsymbol{q}<\lim _{k \rightarrow \infty} \overline{\boldsymbol{q}}^{(k)}<\tilde{\boldsymbol{q}}$. 


\section{Sufficient conditions for the convergence of $\left\{\bar{q}^{(k)}\right\}$ to $q$}

In this section, we assume that the sequence of replacement distributions $\left\{\boldsymbol{\alpha}^{(k)}\right\}$ satisfies a property slightly more general than tightness, that is,

Assumption 1. There exist constants $N_{1}, N_{2} \geq 1$ and $a>0$, all independent of $k$, such that

$$
\sum_{i=1}^{\min \left\{N_{1}, k\right\}} \alpha_{i}^{(k)} \geq a \quad \text { for all } k \geq N_{2}
$$

Situations where Assumption 1 fails to hold include $\boldsymbol{\alpha}^{(k)}=\boldsymbol{e}_{k}$ and $\boldsymbol{\alpha}^{(k)}=$ $1 / k$. These special cases will be considered in Section 6 . Replacement with a fixed type, however, satisfies Assumption 1; for example, when $\boldsymbol{\alpha}^{(k)}=\boldsymbol{e}_{1}$, it holds with $N_{1}=1, N_{2}=1$ and $a=1$. An example of sequence of replacement distributions satisfying Assumption 1 but which is not tight is $\boldsymbol{\alpha}^{(k)}=(a, 0, \ldots, 0,1-a)$ for some $0<a<1$.

\subsection{A motivating example}

In the next example we demonstrate that Assumption 1 alone is not sufficient to ensure $\overline{\boldsymbol{q}}^{(k)} \rightarrow \boldsymbol{q}$ as $k \rightarrow \infty$.

Example 1. Consider a two-parameter irreducible branching process $\left\{\boldsymbol{Z}_{n}\right\}$ with countably many types where, at death, type- 1 individuals produce a single type-2 individual with probability $a>0$ and no offspring with probability $1-a$, and each type- $i \in\{2,3, \ldots\}$ individual produces a single type- $(i+1)$ offspring with probability one and a further Poisson $\left(b^{i-1}\right)$ type-1 individuals, where $0<b<1$. The progeny generating function of this process is

$$
G_{1}(s)=a s_{2}+1-a,
$$

and for $i \geq 2$,

$$
G_{i}(\boldsymbol{s})=\sum_{k \geq 0} \frac{\left(b^{i-1}\right)^{k}}{k !} e^{-b^{i-1}} s_{1}^{k} s_{i+1}=\exp \left\{b^{i-1}\left(s_{1}-1\right)\right\} s_{i+1} .
$$

The corresponding mean progeny representation graph is shown in Figure 4.1. 


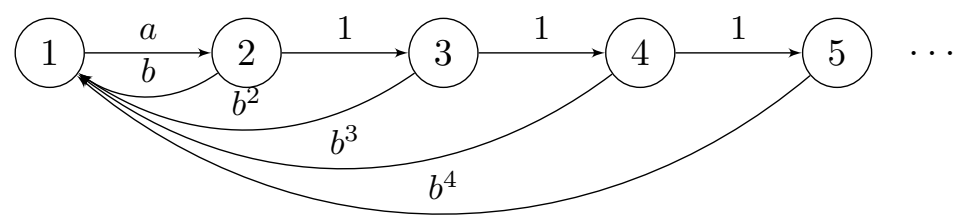

Figure 4.1: The mean progeny representation graph corresponding to Example 1.

From the definition of the process it is clear that the global extinction probability vector $\boldsymbol{q}$ contains entries $q_{1}=1-a$ and $q_{i}=0$ for all $i \geq 2$. We assume that the population initially contains a single individual of type 1 . Because $\left\{\boldsymbol{Z}_{n}\right\}$ is irreducible, the probability of partial extinction $\tilde{q}_{1}$ is equal to the extinction probability of the embedded type-1 process $\left\{E_{n}^{(1)}(\boldsymbol{Z})\right\}$. We observe with the help of Figure 4.1 that in this embedded process individuals have no offspring with probability $1-a$, otherwise they give birth according to the sum of countably infinitely many independent Poisson random variables with respective means $b^{i-1}$, for $i \geq 2$. Consequently, $\tilde{q}_{1}$ is the minimal nonnegative solution of

$$
x=1-a+a F(x), \quad \text { for } 0 \leq x \leq 1,
$$

where $F(\cdot)$ is given by

$$
F(x)=\prod_{i \geq 2}^{\infty} \exp \left\{b^{i-1}(x-1)\right\}=\exp \left\{b(1-b)^{-1}(x-1)\right\} .
$$

Note that $F(\cdot)$ is the probability generating function of a Poisson random variable with mean $b /(1-b)$. The corresponding mean progeny $m_{E_{n}^{(1)}(\boldsymbol{Z})}=$ $a b /(1-b)$ indicates that $\tilde{q}_{1}=1$ if and only if $a \leq(1-b) / b$, in which particular cases $1-a=q_{1}<\tilde{q}_{1}$. The left panel in Figure 4.2 shows the difference $\tilde{q}_{1}-q_{1}$ as a function of the parameter values.

Now consider the process $\left\{\overline{\boldsymbol{Z}}_{n}^{(k)}\right\}$ for $\boldsymbol{\alpha}^{(k)}=\boldsymbol{e}_{1}$, and its conditional extinction probability $\bar{q}_{1}^{(k)}$, given that $\varphi_{0}=1$. This irreducible branching process has finitely many types, hence it becomes extinct if and only if type 1 becomes extinct. Thus, $\bar{q}_{1}^{(k)}$ corresponds to the extinction probability of the type-1 process embedded with respect to $\left\{\overline{\boldsymbol{Z}}_{n}^{(k)}\right\}$. The progeny generating function of $\left\{E_{n}^{(1)}\left(\overline{\boldsymbol{Z}}^{(k)}\right)\right\}$, that we denote by $G_{1, k}(\cdot)$, is given by

$$
G_{1, k}(x)=1-a+a F_{k}(x) x, \quad \text { for } 0 \leq x \leq 1,
$$



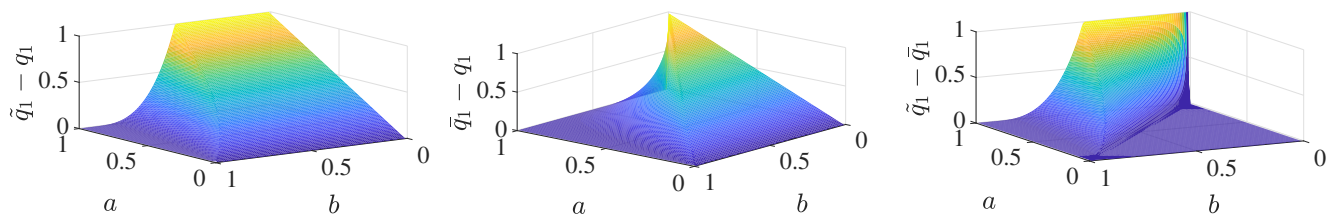

Figure 4.2: Differences in the extinction probabilities related to Example 1, plotted as a function of the parameters $a$ and $b$.

where

$$
F_{k}(x)=\prod_{i \geq 2}^{k} \exp \left\{b^{i-1}(x-1)\right\}=\exp \left\{b\left(1-b^{k-1}\right)(1-b)^{-1}(x-1)\right\}
$$

is the p.g.f of a Poisson random variable with parameter $b\left(1-b^{k-1}\right)(1-b)^{-1}$. Note that here we multiply $a F_{k}(x)$ by $x$ to account for the type- $(k+1)$ descendant (instantaneously replaced by type 1 ) of each type-1 individual that has a type- 2 offspring. By continuity of $G_{1, k}(\cdot)$, the limit $\bar{q}_{1}:=\lim _{k \rightarrow \infty} \bar{q}_{1}^{(k)}$ is the minimal non-negative solution of

$$
x=1-a+a F(x) x,
$$

where $F(x)$ is given in (4.1). The corresponding mean progeny $m_{1, \infty}=$ $G_{1, \infty}^{\prime}(1)=a(1+b /(1-b))=a+m_{E_{n}^{(1)}(\boldsymbol{Z})}$ indicates that $\bar{q}_{1}=1$ if and only if $a \leq 1-b$, in which particular cases $\bar{q}_{1}>q_{1}=1-a$. The middle panel in Figure 4.2 shows the difference $\bar{q}_{1}-q_{1}$ as a function of the parameter values. This highlights the fact that the sequence $\left\{\overline{\boldsymbol{q}}^{(k)}\right\}$ does not always converge to the global extinction probability $\boldsymbol{q}$.

For completeness, we also provide in the right panel of Figure 4.2 the difference $\tilde{q}_{1}-\bar{q}_{1}$ as a function of the parameter values. From above, we have that if $a \leq 1-b$ then $q_{1}<\tilde{q}_{1}=\bar{q}_{1}=1$, so the sequence $\left\{\overline{\boldsymbol{q}}^{(k)}\right\}$ can potentially converge to the partial extinction probability. 


\subsection{The seed process}

Example 1 illustrates the need to further explore the conditions under which $\left\{\overline{\boldsymbol{q}}^{(k)}\right\}$ converges to $\boldsymbol{q}$ as $k \rightarrow \infty$. Observe that, for any $k \geq 1$, we have

$$
\begin{aligned}
\bar{q}_{i}^{(k)}= & \mathbf{P}_{i}\left(\lim _{n \rightarrow \infty}\left|\overline{\boldsymbol{Z}}_{n}^{(k)}\right|=0\right) \\
= & \mathbf{P}_{i}\left(\lim _{n \rightarrow \infty}\left|\overline{\boldsymbol{Z}}_{n}^{(k)}\right|=0, \lim _{n \rightarrow \infty}\left|\boldsymbol{Z}_{n}^{(k)}\right|=0\right) \\
& \quad+\mathbf{P}_{i}\left(\lim _{n \rightarrow \infty}\left|\overline{\boldsymbol{Z}}_{n}^{(k)}\right|=0, \liminf _{n \rightarrow \infty}\left|\boldsymbol{Z}_{n}^{(k)}\right|>0\right) \\
= & q_{i}^{(k)}+\sum_{x \geq 1}\left(\boldsymbol{\alpha}^{(k)} \overline{\boldsymbol{q}}^{(k)}\right)^{x} \mathbf{P}_{i}\left(\left|\boldsymbol{S}_{k}\right|=x\right)
\end{aligned}
$$

where $\left|\boldsymbol{S}_{k}\right|$ denotes the (finite) number of sterile types produced over the lifetime of $\left\{\tilde{\boldsymbol{Z}}_{n}^{(k)}\right\}$, which are replaced by some random types in $\left\{\overline{\boldsymbol{Z}}_{n}^{(k)}\right\}$ and immortal types in $\left\{\boldsymbol{Z}_{n}^{(k)}\right\}$. To understand Equation (4.2) one may think of simulating the branching processes with $\varphi_{0}=i$ in two stages: by first constructing the path of $\left\{\tilde{\boldsymbol{Z}}_{n}^{(k)}\right\}$, and then constructing those of $\left\{\boldsymbol{Z}_{n}^{(k)}\right\}$ and $\left\{\overline{\boldsymbol{Z}}_{n}^{(k)}\right\}$ by taking the outcome of $\left\{\tilde{\boldsymbol{Z}}_{n}^{(k)}\right\}$, replacing the sterile individuals, and simulating their daughter processes according to the respective replacement and updating rules. Conditional on the first stage of simulation, there are two ways in which $\left\{\overline{\boldsymbol{Z}}_{n}^{(k)}\right\}$ can die, either: $(i)\left\{\tilde{\boldsymbol{Z}}_{n}^{(k)}\right\}$ dies before producing a sterile type, in which case $\left\{\boldsymbol{Z}_{n}^{(k)}\right\}$ also dies (this occurs with probability $\left.q_{i}^{(k)}\right)$, or $(i i)\left\{\tilde{\boldsymbol{Z}}_{n}^{(k)}\right\}$ dies after producing $1 \leq x<\infty$ sterile individuals, in which case $\left\{\overline{\boldsymbol{Z}}_{n}^{(k)}\right\}$ dies with probability $\left(\boldsymbol{\alpha}^{(k)} \overline{\boldsymbol{q}}^{(k)}\right)^{x}$ in the second stage of simulation.

Because $\boldsymbol{q}^{(k)} \rightarrow \boldsymbol{q}$, this generally indicates that in order for $\left\{\overline{\boldsymbol{q}}^{(k)}\right\}$ to converge to $\boldsymbol{q}$, we need to avoid cases where there is a positive probability that the number of sterile individuals produced over the lifetime of $\left\{\tilde{\boldsymbol{Z}}_{n}^{(k)}\right\}$ remains positive and uniformly bounded for all $k$. This is not satisfied in Example 1 as, for any $a<1,0<b<1$ and for all $k \geq 1$,

$$
\mathbf{P}_{1}\left(\left|\boldsymbol{S}_{k}\right|=1\right)>a F(0)>0 .
$$

We defer a formal statement of this idea until Lemma 3 and now formally introduce the seed process $\left\{\boldsymbol{S}_{k}\right\}_{k \geq 0}$, defined from the paths of $\left\{\tilde{\boldsymbol{Z}}_{n}^{(k)}\right\}$.

Definition 1. The seed process $\left\{\boldsymbol{S}_{k}=\left(S_{k, 1}, S_{k, 2}, \ldots\right)\right\}_{k \geq 0}$ defined on $(\Omega, \mathcal{F}, \mathbf{P})$ is such that for any $\omega \in \Omega, \boldsymbol{S}_{0}(\omega)=\boldsymbol{Z}_{0}(\omega)$, and for $k \geq 1$, if $\lim _{n \rightarrow \infty} \tilde{\boldsymbol{Z}}_{n}^{(k)}(\omega)=$ 

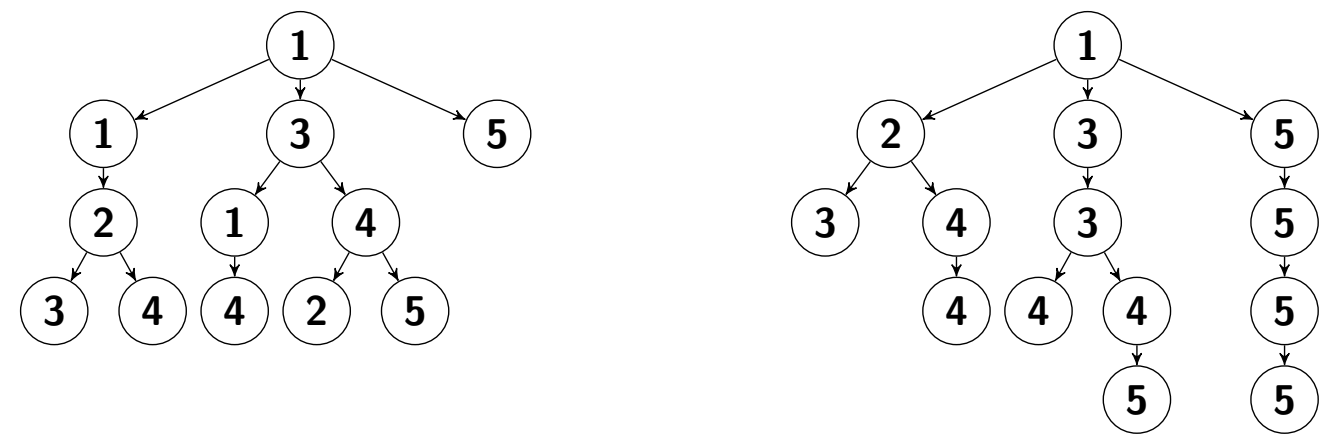

Figure 4.3: A realisation of $\left\{\boldsymbol{Z}_{n}\right\}$ (left). The corresponding seed process, as introduced in Definition 1, is $\boldsymbol{S}_{0}=(1,0,0, \ldots), \boldsymbol{S}_{1}=(0,1,1,0,1, \ldots), \boldsymbol{S}_{2}=(0,0,2,1,1, \ldots)$, $\boldsymbol{S}_{3}=(0,0,0,3,1, \ldots), \boldsymbol{S}_{4}=(0,0,0,0,2, \ldots)$ and $\boldsymbol{S}_{5}=(0,0,0,0,0, \ldots)$. The right graph illustrates the tree representation of $\left\{\boldsymbol{S}_{k}\right\}$.

$\mathbf{0}$, then

$$
S_{k, i}(\omega)= \begin{cases}0, & \text { if } i \leq k, \\ \sum_{n=1}^{\infty} \tilde{Z}_{n, i}^{(k)}(\omega), & \text { if } i>k\end{cases}
$$

otherwise,

$$
S_{k, i}(\omega)=0 \quad \text { for all } i \geq 1
$$

In order to ensure that $\mathbf{P}_{i}\left(\left|\boldsymbol{S}_{k}\right|<\infty\right)=1$ for all fixed $i$ and $k$, we make the convention that $\left|\boldsymbol{S}_{k}(\omega)\right|=0$ when $\left\{\tilde{\boldsymbol{Z}}_{n}^{(k)}(\omega)\right\}$ does not become extinct for some $\omega \in \Omega$. We say that the seed process undergoes a total catastrophe at generation $k$ if $\left\{\tilde{\boldsymbol{Z}}_{n}^{(k-1)}(\omega)\right\}$ becomes extinct while $\left\{\tilde{\boldsymbol{Z}}_{n}^{(k)}(\omega)\right\}$ survives. Since $\left\{\omega \in \Omega: \lim _{n \rightarrow \infty} \tilde{\boldsymbol{Z}}_{n}^{(k)}=\mathbf{0}\right\} \subseteq\left\{\omega \in \Omega: \lim _{n \rightarrow \infty} \tilde{\boldsymbol{Z}}_{n}^{(k-1)}=\mathbf{0}\right\}$ for all $k$, once the seed process experiences total catastrophe it cannot recover.

Each sterile individual of type $i>k$ that appears during the lifetime of $\left\{\tilde{\boldsymbol{Z}}_{n}^{(k)}(\omega)\right\}$ either corresponds to a sterile individual of type $i>k$ that appears during the lifetime of $\left\{\tilde{\boldsymbol{Z}}_{n}^{(k-1)}(\omega)\right\}$, or is a descendant of a sterile individual of type $i=k$ that appears during the lifetime of $\left\{\tilde{\boldsymbol{Z}}_{n}^{(k-1)}(\omega)\right\}$. Consequently, for a given $\omega \in \Omega$, the sequence $\left\{\boldsymbol{S}_{k}(\omega)\right\}$ admits a unique tree representation that can be constructed from the tree of $\left\{\boldsymbol{Z}_{n}(\omega)\right\}$. This is illustrated in Figure 4.3. The nodes of the tree of the seed process will be called seeds. Because the daughter process of the individuals of type $i>k-1$ in $\left\{\tilde{\boldsymbol{Z}}_{n}^{(k)}(\omega)\right\}$ are independent and identically distributed, the process $\left\{\boldsymbol{S}_{k}\right\}_{k \geq 1}$ satisfies the 
recursive equation

$$
\boldsymbol{S}_{k} \stackrel{d}{=}\left(\sum_{i=k}^{\infty} \sum_{j=1}^{S_{k-1, i}} \boldsymbol{\xi}_{i, j}^{(k)}\right) \mathbf{1}\left\{\left(\sum_{i=k}^{\infty} \sum_{j=1}^{S_{k-1, i}} \boldsymbol{\xi}_{i, j}^{(k)}\right)<\infty\right\},
$$

where for any type $i,\left\{\boldsymbol{\xi}_{i, j}^{(k)}\right\}_{j \geq 0}$ is a sequence of i.i.d. random variables such that

$$
\mathbf{P}\left(\boldsymbol{\xi}_{i, j}^{(k)}=\boldsymbol{x}\right)=\mathbf{P}_{i}\left(\left(\sum_{n \geq 0} \sum_{\ell>k} \tilde{Z}_{n, \ell}^{(k)} \boldsymbol{e}_{\ell}\right)=\boldsymbol{x}\right)
$$

for any infinite vector $\boldsymbol{x}$ whose first $k$ entries are 0 and other entries take nonnegative integer values. This implies that $\left\{\boldsymbol{S}_{k}\right\}$ is a branching process with countably many types, whose progeny distribution depends on the generation (branching process in varying environment), and which can experience total catastrophe.

The seed process enjoys several properties which will be exploited to prove Theorem 1 on the convergence of $\left\{\overline{\boldsymbol{q}}^{(k)}\right\}$ to $\boldsymbol{q}$ stated in the next subsection.

Lemma 2. $\mathbf{P}_{i}\left(\left|\boldsymbol{S}_{k}\right|=0\right)=1-\tilde{q}_{i}^{(k)}+q_{i}^{(k)}$.

Proof. By construction of the seed process,

$$
\mathbf{P}_{i}\left(\left|\boldsymbol{S}_{k}\right|=0\right)=\mathbf{P}_{i}\left(\left\{\liminf _{n \rightarrow \infty}\left|\tilde{\boldsymbol{Z}}_{n}^{(k)}\right|>0\right\} \cup\left\{\lim _{n \rightarrow \infty}\left|\boldsymbol{Z}_{n}^{(k)}\right|=0\right\}\right),
$$

where the two events are mutually exclusive.

This provides us with a condition for the global and partial extinction probabilities to coincide:

Corollary 2. For all $i \geq 1$, the following two statements are equivalent

(i) $q_{i}=\tilde{q}_{i}$

(ii) $\mathbf{P}_{i}\left(\lim _{k \rightarrow \infty}\left|\boldsymbol{S}_{k}\right|=0\right)=1$.

We rewrite equation (4.2) as

$$
\bar{q}_{i}^{(k)}-q_{i}^{(k)}=\mathbf{E}_{i}\left(\left(\boldsymbol{\alpha}^{(k)} \overline{\boldsymbol{q}}^{(k)}\right)^{\left|\boldsymbol{S}_{k}\right|}-\mathbf{1}\left\{\left|\boldsymbol{S}_{k}\right|=0\right\}\right) .
$$

The next lemma formalises the discussion preceding Definition 1. 
Lemma 3. Assume that, for some $i \in \mathcal{S}$, there exists $B<\infty$ such that

$$
\liminf _{k \rightarrow \infty} \mathbf{P}_{i}\left(0<\left|\boldsymbol{S}_{k}\right|<B\right)=\pi,
$$

for some $\pi>0$. If, in addition, $\left\{\boldsymbol{\alpha}^{(k)}\right\}$ satisfies Assumption 1 for some $N_{1}$ such that $q_{j}>0$ for all $j \in\left\{1, \ldots, N_{1}\right\}$, then $\liminf _{k \rightarrow \infty} \bar{q}_{i}^{(k)}>q_{i}$.

Proof. Since $q_{j}^{(k)} \rightarrow q_{j}>0$ for all $j \in\left\{1, \ldots, N_{1}\right\}$, there exists $\beta>0$ and $K \in \mathbb{N}$ such that, for all $k>K$ and $j \in\left\{1, \ldots, N_{1}\right\}, q_{j}^{(k)} \geq \beta$. By Lemma 1 , we also have $\overline{\boldsymbol{q}}^{(k)} \geq \boldsymbol{q}^{(k)}$ for all $k \in \mathbb{N}$. Hence

$$
\bar{q}_{j}^{(k)} \geq \beta \text { for all } k>K \text { and all } j \in\left\{1, \ldots, N_{1}\right\} .
$$

It follows from Assumption 1 that for any $k>\max \left\{K, N_{1}, N_{2}\right\}$,

$$
\boldsymbol{\alpha}^{(k)} \overline{\boldsymbol{q}}^{(k)} \geq \sum_{j=1}^{N_{1}} \alpha_{j}^{(k)} \bar{q}_{j}^{(k)} \geq \beta a>0 .
$$

Then, by (4.6),

$$
\begin{aligned}
& \liminf _{k \rightarrow \infty}\left(\bar{q}_{i}^{(k)}-q_{i}^{(k)}\right) \\
& \quad=\liminf _{k \rightarrow \infty} \mathbf{E}_{i}\left(\left(\boldsymbol{\alpha}^{(k)} \overline{\boldsymbol{q}}^{(k)}\right)^{\left|\boldsymbol{S}_{k}\right|}-\mathbf{1}\left\{\left|\boldsymbol{S}_{k}\right|=0\right\}\right) \\
& \geq \liminf _{k \rightarrow \infty} \mathbf{E}_{i}\left(\left(\boldsymbol{\alpha}^{(k)} \overline{\boldsymbol{q}}^{(k)}\right)^{\left|\boldsymbol{S}_{k}\right|}|0<| \boldsymbol{S}_{k} \mid<B\right) \mathbf{P}_{i}\left(0<\left|\boldsymbol{S}_{k}\right|<B\right) \\
& \geq(\beta a)^{B} \pi>0,
\end{aligned}
$$

which completes the proof.

Lemma 3 suggests that the conditions we impose for $\overline{\boldsymbol{q}}^{(k)} \rightarrow \boldsymbol{q}$ should also be sufficient for $\left\{\left|\boldsymbol{S}_{k}\right|\right\}$ to satisfy the dichotomy between extinction and unbounded growth, that is, either there exists an integer $n(\omega)$ for which $\left|\boldsymbol{S}_{k}(\omega)\right|=0$ for all $k \geq n(\omega)$, or $\left|\boldsymbol{S}_{k}(\omega)\right| \rightarrow \infty$ as $k \rightarrow \infty$, for all $\omega \in A$ with $\mathbf{P}(A)=1$. We impose a similar but more general assumption than the well known sufficient condition ' $\inf _{i} q_{i}>0$ ' for $\left\{\left|\boldsymbol{Z}_{n}\right|\right\}$ to satisfy the dichotomy property (see $[20,14])$ :

Assumption 2. $\liminf _{i} q_{i}>0$. 
Assumption 2 is satisfied in particular when $\liminf _{i} p_{i}(\mathbf{0})>0$.

Lemma 4. Suppose Assumption 2 holds, then for all $i \in \mathcal{S}$

$$
\mathbf{P}_{i}\left(\left|\boldsymbol{S}_{k}\right| \rightarrow 0 \text { or } \infty\right)=1 \text {. }
$$

Proof. By Assumption 2 there exist $N_{3} \in \mathbb{N}$ and $\beta>0$ such that $q_{i}>\beta$ for all $i>N_{3}$, and by Lemma $2, \mathbf{P}_{i}\left(\lim _{k \rightarrow \infty}\left|\boldsymbol{S}_{k}\right|=0\right) \geq q_{i}$ for all $i$. Thus for all $1 \leq i \leq N_{3}$

$$
\mathbf{P}_{i}\left(\lim _{k \rightarrow \infty}\left|\boldsymbol{S}_{k}\right|=0\right) \geq \mathbf{E}_{i}\left(\prod_{j=1}^{\infty} q_{j}^{S_{N_{3}, j}}\right) \geq \mathbf{E}_{i}\left(\beta^{\left|\boldsymbol{S}_{N_{3}}\right|}\right):=\delta_{i}>0
$$

and for all $i>N_{3}, \mathbf{P}_{i}\left(\lim _{k \rightarrow \infty}\left|\boldsymbol{S}_{k}\right|=0\right) \geq \beta$. Up to the possibility of a total catastrophe, the individuals in $\left\{\boldsymbol{S}_{k}\right\}$ behave independently, hence for any $\left|\boldsymbol{s}_{\mathbf{0}}\right| \leq x$, we have

$$
\mathbf{P}\left(\lim _{k \rightarrow \infty}\left|\boldsymbol{S}_{k}\right|=0 \mid \boldsymbol{S}_{0}=\boldsymbol{s}_{0}\right) \geq\left(\min \left\{\inf _{1 \leq i \leq N_{3}} \delta_{i}, \beta\right\}\right)^{x}>0 .
$$

Combining Equation (4.7) with the fact that $\left\{\boldsymbol{S}_{k}\right\}$ is a Markov chain with absorbing state $\mathbf{0}$, the result then follows from [20, Theorem 2].

In specific cases, the extinction probability of the seed process can be easier to analyse than that of the original branching process. In [8] we consider one such subclass of branching processes called lower Hessenberg where, by building upon the results of the present section, we are able to analyse the set of fixed points of the original process and derive necessary and sufficient conditions for its almost sure global extinction.

\subsection{Convergence to global extinction}

In this section, we state our result on the pointwise convergence of the sequence $\left\{\overline{\boldsymbol{q}}^{(k)}\right\}$ to the global extinction probability $\boldsymbol{q}$. To obtain convergence, Equation (4.6) suggests that, conditional on $\mathbf{P}\left(\left|\boldsymbol{S}_{k}\right| \rightarrow \infty\right)>0$, one must show that $\boldsymbol{\alpha}^{(k)} \overline{\boldsymbol{q}}^{(k)}$ is bounded away from 1 for all sufficiently large $k$. To prove this, we use a regenerative argument, which may break down for some replacement distributions $\boldsymbol{\alpha}^{(k)}$, such as the ones presented in the next section. 
For a fixed $k$, each seed in $\boldsymbol{S}_{k}$ corresponds to a sterile individual produced over the lifetime of $\left\{\tilde{\boldsymbol{Z}}_{n}^{(k)}\right\}$. To obtain $\left\{\overline{\boldsymbol{Z}}_{n}^{(k)}\right\}$, these seeds are replaced, independently of each other, by new individuals whose types follow the distribution $\boldsymbol{\alpha}^{(k)}$, and whose daughter processes themselves may be thought of as producing an i.i.d. number of new seeds, and so on. Thus, the process formed by taking all 'replaced' individuals from $\left\{\overline{\boldsymbol{Z}}_{n}^{(k)}\right\}$ which correspond to seeds, and connecting each of these individuals to its nearest replaced seed ancestor in $\left\{\overline{\boldsymbol{Z}}_{n}^{(k)}\right\}$, is a multitype Galton-Watson process on $(\Omega, \mathcal{F}, \mathbf{P})$ with type space $T_{k}=\{1, \ldots, k\}$. We refer to this process as the embedded replacement process, and denote it as $\left\{\boldsymbol{Z}_{n}^{(e, k)}\right\}_{n \geq 0}$. In $\left\{\boldsymbol{Z}_{n}^{(e, k)}\right\}$, each child's type is chosen independently of the type of its parent and other siblings, and therefore the corresponding progeny generating function $\boldsymbol{G}^{(e, k)}:[0,1]^{T_{k}} \rightarrow[0,1]^{T_{k}}$ is such that

$$
G_{i}^{(e, k)}(\boldsymbol{s})=\sum_{x \geq 0}\left(\boldsymbol{\alpha}^{(k)} \boldsymbol{s}\right)^{x} \mathbf{P}_{i}\left(\left|\boldsymbol{S}_{k}\right|=x\right), \quad i \in T_{k} .
$$

We use the convention that $\boldsymbol{Z}_{0}^{(e, k)} \equiv \overline{\boldsymbol{Z}}_{0}^{(k)}$, that is, we include the initial individual in $\boldsymbol{Z}_{0}^{(e, k)}$ regardless of whether it has been replaced. The embedded replacement process can be constructed pathwise for each $\omega \in \Omega$, but we omit the details here. Conditional on the initial type $\varphi_{0} \in T_{k}$, for each $\omega \in \Omega$ we have $\left|\boldsymbol{Z}_{1}^{(e, k)}(\omega)\right|=\left|\boldsymbol{S}_{k}(\omega)\right|$. Figure 4.4 gives an illustration of the construction of $\left\{\boldsymbol{Z}_{n}^{(e, k)}\right\}$ when compared to the corresponding realisation of $\left\{\overline{\boldsymbol{Z}}_{n}^{(k)}\right\}$ when $k=4$ and $\boldsymbol{\alpha}^{(4)}=\boldsymbol{e}_{1}$. In Figure 4.4, the type-2 root is common to both processes and the black type-1 nodes represent individuals that have been replaced.

It is clear that if the embedded replacement process does not become extinct then neither does $\left\{\overline{\boldsymbol{Z}}_{n}^{(k)}\right\}$. Thus,

$$
\bar{q}_{j}^{(k)} \leq q_{j}^{(e, k)} \quad \text { for all } j \in T_{k}
$$

where $\boldsymbol{q}^{(e, k)}$ is the extinction probability vector of $\left\{\boldsymbol{Z}_{n}^{(e, k)}\right\}$. We are now in a position to prove our result on the pointwise convergence of the sequence $\left\{\overline{\boldsymbol{q}}^{(k)}\right\}$ to the global extinction probability $\boldsymbol{q}$.

Theorem 1. Suppose Assumption 2 holds. In addition, suppose that the replacement distributions $\left\{\boldsymbol{\alpha}^{(k)}\right\}$ satisfy Assumption 1 with $N_{1}$ such that either

(i) $\tilde{q}_{j}<1$ for all $j \in\left\{1, \ldots, N_{1}\right\}$, or 

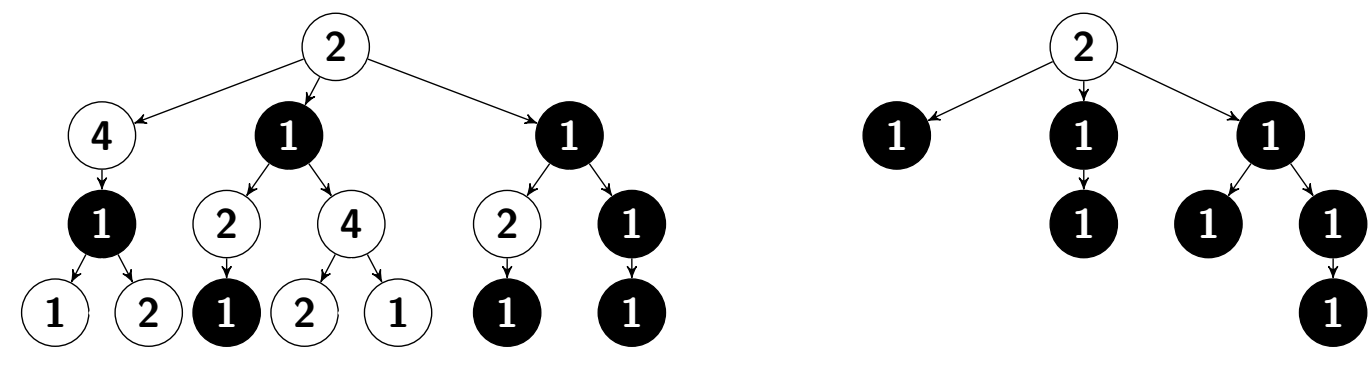

Figure 4.4: A realisation of $\left\{\overline{\boldsymbol{Z}}_{n}^{(4)}\right\}$ (left) with the corresponding realisation of the $\left\{\boldsymbol{Z}_{n}^{(e, 4)}\right\}$ (right) when $\boldsymbol{\alpha}^{(k)}=\boldsymbol{e}_{1}$. The black nodes represent individuals with type greater than 4 which are immediately replaced with type 1 .

(ii) $\tilde{q}_{j}=1$ for all $j \in\left\{1, \ldots, N_{1}\right\}$, and there is a path from any $j \in$ $\left\{1, \ldots, N_{1}\right\}$ to the initial type $i$.

Then

$$
\lim _{k \rightarrow \infty} \bar{q}_{i}^{(k)}=q_{i} .
$$

In particular, if $\left\{\boldsymbol{Z}_{n}\right\}$ is irreducible, then under Assumptions 1 and 2,

$$
\lim _{k \rightarrow \infty} \overline{\boldsymbol{q}}^{(k)}=\boldsymbol{q} .
$$

If $\left\{\boldsymbol{Z}_{n}\right\}$ is irreducible, then (i) or (ii) immediately follows, but the converse is not true. Theorem 1 therefore holds in many reducible cases too. The conditions on $\tilde{q}_{j}$ are easy to verify since a simple criterion exists for partial extinction, see [16].

Proof. By (4.6), we have for any fixed $i \geq 1, k \geq 1$, and for any arbitrary integer $K \geq 1$,

$$
\begin{aligned}
& \bar{q}_{i}^{(k)}-q_{i}^{(k)}= \\
& \mathbf{E}_{i}\left(\left(\boldsymbol{\alpha}^{(k)} \overline{\boldsymbol{q}}^{(k)}\right)^{\left|\boldsymbol{S}_{k}\right|}|0<| \boldsymbol{S}_{k} \mid<K\right) \mathbf{P}_{i}\left(0<\left|\boldsymbol{S}_{k}\right|<K\right) \\
& \quad+\mathbf{E}_{i}\left(\left(\boldsymbol{\alpha}^{(k)} \overline{\boldsymbol{q}}^{(k)}\right)^{\left|\boldsymbol{S}_{k}\right|}|| \boldsymbol{S}_{k} \mid \geq K\right) \mathbf{P}_{i}\left(\left|\boldsymbol{S}_{k}\right| \geq K\right) .
\end{aligned}
$$

Under Assumption 2, by Lemma 4 we get that for any $K \geq 1, \mathbf{P}_{i}\left(0<\left|\boldsymbol{S}_{k}\right|<\right.$ $K) \rightarrow 0$ as $k \rightarrow \infty$, so (4.10) vanishes as $k \rightarrow \infty$. It remains to show that (4.11) vanishes as well. 
By Lemmas 2 and $4, \lim _{k \rightarrow \infty} \mathbf{P}_{i}\left(\left|\boldsymbol{S}_{k}\right| \geq K\right)=c_{i}$ independently of the choice of $K$, where $c_{i}=\tilde{q}_{i}-q_{i}$. Thus we obtain

$$
\limsup _{k \rightarrow \infty}\left(\bar{q}_{i}^{(k)}-q_{i}^{(k)}\right) \leq c_{i} \limsup _{k \rightarrow \infty}\left(\boldsymbol{\alpha}^{(k)} \overline{\boldsymbol{q}}^{(k)}\right)^{K}
$$

We prove next that $\boldsymbol{\alpha}^{(k)} \overline{\boldsymbol{q}}^{(k)}$ is bounded away from 1 for $k$ sufficiently large whenever $c_{i}>0$, assuming (i) and (ii) separately.

Assume that (i) holds. Then there exists $\varepsilon>0$ and $L \geq 1$ such that for all $j \in\left\{1, \ldots, N_{1}\right\}$, and for all $k \geq L, \tilde{q}_{j}^{(k)}<1-\varepsilon$. Therefore, by Lemma 1 , for all $j \in\left\{1, \ldots, N_{1}\right\}$ and $k \geq L$, we have $\bar{q}_{j}^{(k)}<1-\varepsilon$. It follows that for any $k>\max \left\{L, N_{1}\right\}$,

$$
\begin{aligned}
\boldsymbol{\alpha}^{(k)} \overline{\boldsymbol{q}}^{(k)} & =\sum_{j=1}^{N_{1}} \alpha_{j}^{(k)} \bar{q}_{j}^{(k)}+\sum_{j=N_{1}+1}^{k} \alpha_{j}^{(k)} \bar{q}_{j}^{(k)} \\
& <(1-\varepsilon) \sum_{j=1}^{N_{1}} \alpha_{j}^{(k)}+\sum_{j=N_{1}+1}^{k} \alpha_{j}^{(k)} \\
& =\sum_{j=1}^{k} \alpha_{j}^{(k)}-\varepsilon \sum_{j=1}^{N_{1}} \alpha_{j}^{(k)} \leq 1-\varepsilon a,
\end{aligned}
$$

where the last inequality follows from Assumption 1. With this, (4.12) becomes

$$
\limsup _{k \rightarrow \infty}\left(\bar{q}_{i}^{(k)}-q_{i}^{(k)}\right)<c_{i}(1-\varepsilon a)^{K}
$$

and the result follows from Corollary 1 and by choosing $K$ large enough.

Assume that (ii) holds. First observe that if $c_{i}=0$ for all $i \geq 1$ in (4.12), then the result immediately follows. In the remainder of the proof we assume that there exists $i \geq 1$ such that $c_{i}>0$, and we first show that this implies that $c_{j}>0$ for all $j \in\left\{1, \ldots, N_{1}\right\}$. Indeed, let $\theta_{i}$ be the first time an individual of type $i$ is born in $\left\{\boldsymbol{Z}_{n}\right\}$.

Then, by assumption, $\mathbf{P}_{j}\left(\theta_{i}<\infty\right)>0$ for all $j \in\left\{1, \ldots, N_{1}\right\}$. Next, we have

$$
\mathbf{P}_{j}\left(\left|\boldsymbol{S}_{k}\right| \geq K\right) \geq \mathbf{P}_{j}\left(\left|\boldsymbol{S}_{k}\right| \geq K \mid \theta_{i}<\tau_{k}\right) \mathbf{P}_{j}\left(\theta_{i}<\tau_{k}\right) .
$$

In addition, if the process starts with one type- $j$ individual and generates a type- $i$ individual before a seed, then the total number of seeds would be 
larger than $K$ if the type- $i$ individual itself generates more than $K$ seeds, that is,

$$
\mathbf{P}_{i}\left(\left|\boldsymbol{S}_{k}\right| \geq K\right) \leq \mathbf{P}_{j}\left(\left|\boldsymbol{S}_{k}\right| \geq K \mid \theta_{i}<\tau_{k}\right)
$$

so that

$$
\mathbf{P}_{j}\left(\left|\boldsymbol{S}_{k}\right| \geq K\right) \geq \mathbf{P}_{i}\left(\left|\boldsymbol{S}_{k}\right| \geq K\right) \mathbf{P}_{j}\left(\theta_{i}<\tau_{k}\right) .
$$

Note that here we use the assumption $\tilde{q}_{j}=1$ to avoid the possibility of total catastrophe in the seed process. As $k \rightarrow \infty$, the last inequality becomes $c_{j} \geq c_{i} \mathbf{P}_{j}\left(\theta_{i}<\infty\right)>0$, as required.

Now that we have shown $c_{i}>0$ for some $i \geq 1$ implies $c_{j}>0$ for all $j \in\left\{1, \ldots, N_{1}\right\}$, it remains to show that $c_{i}>0$ implies $\boldsymbol{\alpha}^{(k)} \overline{\boldsymbol{q}}^{(k)}$ is bounded away from 1 for $k$ sufficiently large. Since $c_{j}>0$, it follows that for any $\varepsilon>0$ there exists an integer $W_{j}$ depending on $K$ such that for all $k>W_{j}$,

$$
\mathbf{P}_{j}\left(\left|\boldsymbol{S}_{k}\right| \geq K\right)>c_{j}-\varepsilon .
$$

Let $W=\max _{1 \leq j \leq N_{1}}\left\{W_{j}\right\}$, and $c=\min _{1 \leq j \leq N_{1}}\left\{c_{j}\right\}>0$. With reference to (4.8) and (4.13), we observe that for any $k \geq\left\{W, N_{1}, N_{2}\right\}$, the process $\left\{\boldsymbol{Z}_{n}^{(e, k)}: \varphi_{0} \in\left\{1, \ldots, N_{1}\right\}\right\}$ is then stochastically larger than the branching process $\left\{\boldsymbol{Z}_{n}^{(e, k, 2)}\right\}_{n \geq 0}$ with type set $T_{N_{1}}:=\left\{1, \ldots, N_{1}\right\}$ and progeny generating function $\boldsymbol{G}^{(e, k, 2)}:[0,1]^{T_{N_{1}}} \rightarrow[0,1]^{T_{N_{1}}}$ such that, for any $i \in T_{N_{1}}$,

$$
G_{i}^{(e, k, 2)}(\boldsymbol{s})=\left(\sum_{j=1}^{N_{1}} \alpha_{j}^{(k)} s_{j}+1-\sum_{j=1}^{N_{1}} \alpha_{j}^{(k)}\right)^{K}(c-\varepsilon)+1-(c-\varepsilon) .
$$

This corresponds to the branching process in which each individual has $K$ offspring with probability $c-\varepsilon$ and 0 offspring otherwise, then the types of the offspring are assigned independently according to the possibly defective distribution $\left(\alpha_{1}^{(k)}, \ldots, \alpha_{N_{1}}^{(k)}\right)$ and individuals not assigned a type are immediately killed. Since $G_{i}^{(e, k, 2)}(\cdot)$ is independent of $i,\left\{\left|\boldsymbol{Z}_{n}^{(e, k, 2)}\right|\right\}_{n \geq 0}$ behaves like a single-type Galton-Watson process, that is, it is locally isomorphic to a single-type branching process (see [35, Definition 4.2]). Combining this with the fact that by Assumption $1, \sum_{i=1}^{N_{1}} \alpha_{i}^{(k)} \geq a$ for all $k \geq N_{2}$, we see that, for all $k \geq \max \left\{W, N_{1}, N_{2}\right\},\left\{\left|\boldsymbol{Z}_{n}^{(e, k, 2)}\right|\right\}$ is stochastically larger than the single-type branching process $\left\{Z_{n}^{(e, 3)}\right\}_{n \geq 0}$ with progeny generating function

$$
G^{(e, 3)}(s)=(a s+1-a)^{K}(c-\varepsilon)+1-(c-\varepsilon) .
$$


By taking $K>2 /(a(c-\varepsilon))$ in order to bound the mean progeny of $\left\{Z_{n}^{(e, 3)}\right\}$ away from 1 , we obtain $q_{j}^{(e, k)} \leq q_{j}^{(e, k, 2)} \leq q^{(e, 3)}<1-\gamma$ for any $k \geq$ $\max \left\{W, N_{1}, N_{2}\right\}, j \in\left\{1, \ldots, N_{1}\right\}$, and for some $\gamma>0$. Using the same argument as the one used when assuming (i) holds, we obtain $\boldsymbol{\alpha}^{(k)} \boldsymbol{q}^{(e, k)}<1-\gamma a$, and therefore by (4.9),

$$
\boldsymbol{\alpha}^{(k)} \overline{\boldsymbol{q}}^{(k)} \leq \boldsymbol{\alpha}^{(k)} \boldsymbol{q}^{(e, k)}<1-\gamma a
$$

for $k$ sufficiently large, which proves the result.

\section{Conditions for $q<1$ and $q=1$}

Theorem 1 establishes a relationship between extinction of finite-type branching processes and global extinction of infinite-type branching processes. We now directly exploit this link and well-known results on finite-type branching processes in a first attempt to derive sufficient conditions for $\boldsymbol{q}=\mathbf{1}$ and $\boldsymbol{q}<\mathbf{1}$. Throughout this section we assume that $\left\{\boldsymbol{Z}_{n}\right\}$ and $\left\{\boldsymbol{\alpha}^{(k)}\right\}$ satisfy the conditions of Theorem 1.

For $k \geq 1$, let $\tilde{M}^{(k)}$ denote the $k$ th north-west truncation of the mean progeny matrix $M$, and let $\boldsymbol{x}^{(k)}$ be the $k \times 1$ vector such that $x_{i}^{(k)}=\sum_{j>k} M_{i j}$. Then the matrix $\bar{M}^{(k)}:=\tilde{M}^{(k)}+\boldsymbol{x}^{(k)} \boldsymbol{\alpha}^{(k)}$ is the mean progeny matrix of $\left\{\overline{\boldsymbol{Z}}_{n}^{(k)}\right\}$, and $\overline{\boldsymbol{q}}^{(k)}=\mathbf{1}$ if and only if $\rho\left(\bar{M}^{(k)}\right) \leq 1$. This leads to a neat sufficient condition for almost sure global extinction.

Corollary 3. If $\rho\left(\bar{M}^{(k)}\right) \leq 1$ for infinitely many $k$ then $\boldsymbol{q}=\mathbf{1}$.

Corollary 3 implies that if $\liminf _{k} \rho\left(\bar{M}^{(k)}\right)<1$ then $\boldsymbol{q}=\mathbf{1}$. Conversely, one may expect that $\liminf _{k} \rho\left(\bar{M}^{(k)}\right)>1$ implies $\boldsymbol{q}<\mathbf{1}$, however, this is not necessarily the case. Indeed, [35, Example 4.4] corresponds to a case where $\liminf _{k} \rho\left(\bar{M}^{(k)}\right) \geq 2$ and $\boldsymbol{q}=\mathbf{1}$. Additional higher moment conditions are therefore required. We impose the following condition.

Assumption 3. There exists $B_{1}<\infty$ such that $\mathbf{E}_{i}\left(\left|\boldsymbol{Z}_{1}\right|^{2}\right)<B_{1}$ for all $i \geq 0$.

Let $\overline{\boldsymbol{v}}^{(k)}$ denote the right Perron-Frobenius eigenvector of $\bar{M}^{(k)}$ and $\bar{A}_{i, j \ell}^{(k)}:=$ $\partial \bar{G}_{i}^{(k)}(\boldsymbol{s}) /\left.\left(\partial s_{j} \partial s_{\ell}\right)\right|_{s=1}$, for $1 \leq i, j, \ell \leq k$, where $\overline{\boldsymbol{G}}^{(k)}(\boldsymbol{s})$ is the progeny generating function corresponding to $\left\{\overline{\boldsymbol{Z}}_{n}^{(k)}\right\}$. We now provide sufficient conditions for $\boldsymbol{q}<\mathbf{1}$. 
Proposition 1. Under Assumption 3, if $\left\{\boldsymbol{\alpha}^{(k)}\right\}$ is such that

(i) there exists $B_{2}<\infty$ independent of $i, j, k$ such that $\bar{v}_{j}^{(k)} / \bar{v}_{i}^{(k)}<B_{2}$ whenever $\bar{M}_{i j}^{(k)}>0$, and

(ii) there exists $i \geq 1$ such that $\liminf _{k \rightarrow \infty}\left(\bar{v}_{i}^{(k)} / \sup _{j}\left\{\bar{v}_{j}^{(k)}\right\}\right)=b>0$,

then $\liminf _{k} \rho\left(\bar{M}^{(k)}\right)>1$ implies $q_{i}<1$.

Proof. Observe that if there exists $\boldsymbol{s}^{(k)}$ such that $\overline{\boldsymbol{G}}^{(k)}\left(\boldsymbol{s}^{(k)}\right) \leq \boldsymbol{s}^{(k)}$ then $\overline{\boldsymbol{q}}^{(k)} \leq \boldsymbol{s}^{(k)}$. Let $c^{(k)}:=1 / \sup _{j}\left\{\bar{v}_{j}^{(k)}\right\}$. By the Taylor expansion formula in [2, Corollary 3], we have for any $1 \leq i \leq k$ and $0<\theta<1$,

$$
\begin{aligned}
\bar{G}_{i}^{(k)}\left(\mathbf{1}-\theta \overline{\boldsymbol{v}}^{(k)} c^{(k)}\right) & \leq 1-\theta c^{(k)} \sum_{j} \bar{v}_{j}^{(k)} \bar{M}_{i j}^{(k)}+\left(\theta c^{(k)}\right)^{2} \sum_{j, \ell} \bar{v}_{j}^{(k)} \bar{v}_{\ell}^{(k)} \bar{A}_{i, j \ell}^{(k)} \\
& \leq 1-\theta \rho\left(\bar{M}^{(k)}\right) \bar{v}_{i}^{(k)} c^{(k)}+\left(\theta B_{2} \bar{v}_{i}^{(k)} c^{(k)}\right)^{2} \sum_{j, \ell} \bar{A}_{i, j \ell}^{(k)}
\end{aligned}
$$

where $\sum_{j, \ell} \bar{A}_{i, j \ell}^{(k)}=\mathbf{E}_{i}\left(\left|\overline{\boldsymbol{Z}}_{1}^{(k)}\right|^{2}\right)=\mathbf{E}_{i}\left(\left|\boldsymbol{Z}_{1}\right|^{2}\right) \leq B_{1}$. Thus, for any $1<a<$ $\lim \inf \rho\left(\bar{M}^{(k)}\right)$ there exists $K<\infty$ such that

$$
\overline{\boldsymbol{G}}^{(k)}\left(\mathbf{1}-\theta \overline{\boldsymbol{v}}^{(k)} c^{(k)}\right) \leq \mathbf{1}-\theta \overline{\boldsymbol{v}}^{(k)} c^{(k)}\left(a-\theta B_{2}^{2} B_{1}\right),
$$

for all $k>K$. If $\theta<\frac{a-1}{B_{1} B_{2}^{2}}$ then $\overline{\boldsymbol{q}}^{(k)}<\mathbf{1}-\theta \overline{\boldsymbol{v}}^{(k)} c^{(k)}$ for all $k \geq K$. By Theorem 1 and (ii) we then obtain $q_{i}<1-\theta b$.

Observe that if there exists $\varepsilon>0$ such that $\bar{M}_{i j}^{(k)}>0$ implies $\bar{M}_{i j}^{(k)}>\varepsilon$ for all $k \geq 0$, then $\bar{v}_{j}^{(k)} / \bar{v}_{i}^{(k)}<\rho\left(\bar{M}^{(k)}\right) / \varepsilon$. This means that if, in addition, $\lim \sup _{k} \rho\left(\bar{M}^{(k)}\right)<\infty$ then (i) holds. Condition (ii) is generally more difficult to verify. One particular case where it clearly holds is when the row sums of $M$ are constant. It also holds in less trivial examples such as the nearestneighbour branching random walks considered in [16, Section 5.1], but the proof is out of the scope of the paper.

Proposition 1 leads naturally to sufficient conditions for the entries of $\boldsymbol{q}$ to be uniformly bounded away from 1 .

Corollary 4. Under Assumption 3, if $\left\{\boldsymbol{\alpha}^{(k)}\right\}$ is such that $0<b \leq \bar{v}_{i}^{(k)} \leq c<$ $\infty$ for all $k \geq 0$ and $1 \leq i \leq k$, then $\liminf _{k} \rho\left(\bar{M}^{(k)}\right)>1$ implies $\sup _{i} q_{i}<1$. 
Proof. Following the arguments in the proof of Lemma 1, there exists $\theta>0$ such that $q_{i}<1-\theta b / c$ for all $i \geq 1$.

Theorem 4 of [30] is similar to Corollary 4, however it requires the convergence norm $\nu(M)$ of $M$ to be strictly larger than 1, which is known to be sufficient for $\tilde{\boldsymbol{q}}<\mathbf{1}$. Note that repeating the same arguments with the sequence $\left\{\boldsymbol{q}^{(k)}\right\}$ instead of $\left\{\overline{\boldsymbol{q}}^{(k)}\right\}$ leads to a result similar to [30, Theorem 4] since the sequence of spectral radii of the mean progeny matrices corresponding to $\left\{\boldsymbol{Z}_{n}^{(k)}\right\}$ converge to $\max \{1, \nu(M)\}$ as $k \rightarrow \infty$. The primary difference between Corollary 4 and [30, Theorem 4] therefore lies in the conditions ' $\lim \inf _{k} \rho\left(\bar{M}^{(k)}\right)>1$ ' and ' $\nu(M)>1$ '. In both Examples 2 and 3, when $\boldsymbol{q}<\tilde{\boldsymbol{q}}=\mathbf{1}$, the former is satisfied but the latter is not.

\section{Examples and relaxations of Assumption 1}

Theorem 1 proves that $\overline{\boldsymbol{q}}^{(k)} \rightarrow \boldsymbol{q}$ for a large class of replacement distributions $\left\{\boldsymbol{\alpha}^{(k)}\right\}$. In this section we demonstrate that when $\left\{\boldsymbol{\alpha}^{(k)}\right\}$ is chosen so that Assumption 1 does not hold, the sequence $\left\{\overline{\boldsymbol{q}}^{(k)}\right\}$ exhibits a range of asymptotic behaviours. Indeed, we show that its limit does not necessarily exist (Example 2), or does not necessarily converge to $\boldsymbol{q}$ (Example $3)$. The proofs of the results pertaining to these examples are gathered in Appendix B. These results are related to those in $[12,13,17]$, where the algorithmic computation of the stationary distribution of a recurrent infinite state Markov chain was considered.

Example 2 (Replacement with type $k$ ). Let $\boldsymbol{\alpha}^{(k)}=\boldsymbol{e}_{k}$ and consider a modified version of the example of [16, Section 5.1], in which the odd types are 'stronger' than the even types. That is, we assume $a, c>0, d>1$ and define

$$
G_{1}(s)=\frac{c d}{t} s_{2}^{t}+1-\frac{c d}{t}
$$

and for $i \geq 2$,

$$
G_{i}(s)= \begin{cases}\frac{c d}{u} s_{i+1}^{u}+\frac{a d}{u} s_{i-1}^{u}+1-\frac{d(a+c)}{u} & \text { when } i \text { is odd, } \\ \frac{c}{d v} s_{i+1}^{v}+\frac{a}{d v} s_{i-1}^{v}+1-\frac{(a+c)}{d v} & \text { when } i \text { is even, }\end{cases}
$$

where $t=\lceil d c\rceil+1, u=\lceil d(c+a)\rceil+1$ and $v=\lceil(c+a) / d\rceil+1$. 


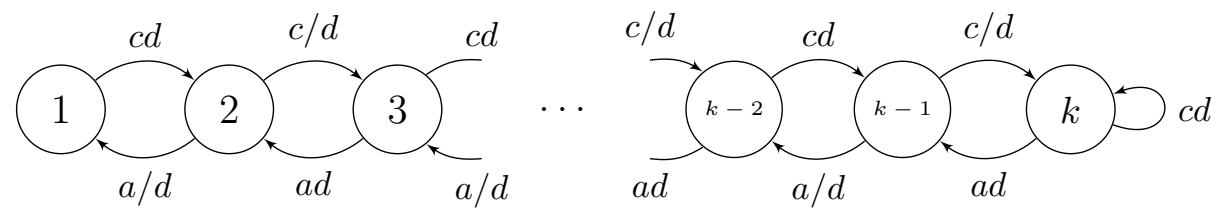

Figure 6.1: The mean progeny representation graph corresponding to $\left\{\overline{\boldsymbol{Z}}_{n}^{(k)}\right\}$ when $k$ is odd in Example 2.

When $k$ is odd $\left\{\overline{\boldsymbol{Z}}_{n}^{(k)}\right\}$ has the mean progeny representation graph given in Figure 6.1; when $k$ is even, there is an equivalent graph. We consider the type- $k$ process $\left\{E_{n}^{(k)}\left(\overline{\boldsymbol{Z}}^{(k)}\right)\right\}$ embedded with respect to $\left\{\overline{\boldsymbol{Z}}_{n}^{(k)}: \varphi_{0}=k\right\}$, with mean progeny $m_{E_{n}^{(k)}\left(\overline{\boldsymbol{Z}}^{(k)}\right)}$ that we denote by $\bar{m}^{(k)}$ for short. The limit of the sequence $\left\{\bar{m}^{(k)}\right\}$ does not generally exist, however its limit superior and inferior are finite when $a c \leq 1 / 4$, as we show in the next lemma.

Lemma 5. The mean progeny of $\left\{E_{n}^{(k)}\left(\overline{\boldsymbol{Z}}^{(k)}\right)\right\}$ described in Example 2 satisfies

$\lim _{k \rightarrow \infty} \bar{m}^{(2 k+1)}=c d+\frac{1}{2}(1-\sqrt{1-4 a c}), \quad \lim _{k \rightarrow \infty} \bar{m}^{(2 k)}=c / d+\frac{1}{2}(1-\sqrt{1-4 a c})$

when $a c \leq 1 / 4$, and $\lim _{k \rightarrow \infty} \bar{m}^{(k)}=+\infty$ when $a c>1 / 4$.

As a consequence of Lemma $5, \lim _{k \rightarrow \infty} \bar{m}^{(2 k+1)}-\bar{m}^{(2 k)}=c\left(d-d^{-1}\right)$, which indicates it is possible to choose $a, c$ and $d$ so that as $k \rightarrow \infty, \bar{m}^{(k)}$ oscillates between values less than 1 and greater than 1 . This observation leads us to the following result.

Proposition 2. Consider the branching process described in Example 2. Assume that $d>1$ and that $\boldsymbol{\alpha}^{(k)}=\boldsymbol{e}_{k}$. Then $\lim _{k \rightarrow \infty} \overline{\boldsymbol{q}}^{(k)}=\boldsymbol{q}$ when ac $>1 / 4$. Additionally, $\tilde{\boldsymbol{q}}=\mathbf{1}$ if and only if ac $\leq 1 / 4$, and when this is satisfied,

(i) if $d^{-1}>(1+\sqrt{1-4 a c}) / 2 c$ then $\lim _{k \rightarrow \infty} \overline{\boldsymbol{q}}^{(k)}=\boldsymbol{q}$,

(ii) if $d^{-1} \leq(1+\sqrt{1-4 a c}) / 2 c<d$ then

$$
\lim _{k \rightarrow \infty} \overline{\boldsymbol{q}}^{(2 k+1)}=\boldsymbol{q} \quad \text { and } \quad \lim _{k \rightarrow \infty} \overline{\boldsymbol{q}}^{(2 k)}=\tilde{\boldsymbol{q}}=\mathbf{1}
$$

(iii) if $d \leq(1+\sqrt{1-4 a c}) / 2 c$ then $\lim _{k \rightarrow \infty} \overline{\boldsymbol{q}}^{(k)}=\tilde{\boldsymbol{q}}=\boldsymbol{q}=\mathbf{1}$. 
In Figure 6.2 we plot $\tilde{q}_{1}^{(k)}$ (black dashed), $q_{1}^{(k)}$ (grey dashed) and $\bar{q}_{1}^{(k)}$ for $\boldsymbol{\alpha}^{(k)}=\boldsymbol{e}_{1}$ (solid grey bold), $\boldsymbol{\alpha}^{(k)}=\mathbf{1} / k$ (solid black bold) and $\boldsymbol{\alpha}^{(k)}=\boldsymbol{e}_{k}$ (solid fine). In the top two plots we let $a=1 / 6$ and $c=7 / 8$, in which case $a c<1 / 4$ and $(1+\sqrt{1-4 a c}) / 2 c \approx 0.94$. With this in mind, we choose $d^{-1}=0.95$ (panel (a)) and $d^{-1}=0.93$ (panel (b)). In agreement with Proposition 2, for $\boldsymbol{\alpha}^{(k)}=\boldsymbol{e}_{k}$ we observe that $\bar{q}_{1}^{(k)} \rightarrow q_{1}$ when $d^{-1}=0.95$, whereas $\bar{q}_{1}^{(2 k+1)} \rightarrow q_{1}$ and $\bar{q}_{1}^{(2 k)} \rightarrow \tilde{q}_{1}=1$ when $d^{-1}=0.93$. For these values of $a, c$ and $d$ it appears that $q_{1}<1$, which lead us to conclude that $\liminf _{k \rightarrow \infty} \bar{q}_{1}^{(k)} \neq \limsup _{k \rightarrow \infty} \bar{q}_{1}^{(k)}$ when $d^{-1}=0.93$ and $\boldsymbol{\alpha}^{(k)}=\boldsymbol{e}_{k}$. In panel (c) of Figure 6.2 we let $a=1 / 3$ and $c=13 / 16$, in which case $a c>1 / 4$ so that $\tilde{q}_{1}<1$ for any value of $d$. We choose $d=2$ and observe that all sequences converge to $q_{1}=\tilde{q}_{1}$. In panel (d) of Figure 6.2 we let $a=1 / 6, c=13 / 16$ and $d=2$, which means $a c<1 / 4$ and $d^{-1}<(1+\sqrt{1-4 a c}) / 2 c \approx 1.03<d$, which entails that for $\boldsymbol{\alpha}^{(k)}=\boldsymbol{e}_{k}$, $\bar{q}_{1}^{(2 k+1)} \rightarrow q_{1}$ and $\bar{q}_{1}^{(2 k)} \rightarrow \tilde{q}_{1}$. However, in this case $\tilde{q}_{1}=q_{1}=1$ and thus the limit of $\bar{q}^{(k)}$ exists.

Observe that for the branching process described in Example 2, Proposition 2 implies that when $\boldsymbol{\alpha}^{(k)}=\boldsymbol{e}_{k}$, then $\liminf _{k \rightarrow \infty} \overline{\boldsymbol{q}}^{(k)}=\boldsymbol{q}$. The next example indicates that when $\boldsymbol{\alpha}^{(k)}=\mathbf{1} / k$ and $\inf _{i} q_{i}>0$, there is not always a subsequence of $\overline{\boldsymbol{q}}^{(k)}$ that converges to $\boldsymbol{q}$.

Example 3 (Replacement with a uniform type). For ease of notation, in this example we use the type set $\mathcal{S}=\{2,3,4, \ldots\}$. Suppose $p, \varepsilon \in(0,1)$ and $3 p \varepsilon^{2}<1$, and consider the following progeny distribution:

$$
G_{2}(s)=p s_{4}^{3}+(1-p),
$$

and for $i \geq 3$,

$$
G_{i}(\boldsymbol{s})=\left\{\begin{array}{cc}
\varepsilon p\left(1-3 p \varepsilon^{i / 2}\right) s_{i-1} s_{2 i}^{3} & \\
+p\left(1-3 p \varepsilon^{i / 2}\right)(1-\varepsilon) s_{2 i}^{3} & \\
+\varepsilon\left(1-p\left(1-3 p \varepsilon^{i / 2}\right)\right) s_{i-1} & \\
\quad+\left(1-\varepsilon-p\left(1-3 p \varepsilon^{i / 2}\right)(1-\varepsilon)\right), & \text { if } i \in\left\{2^{l}\right\}_{l \geq 2}, \\
\varepsilon s_{i-1}+(1-\varepsilon) & \text { otherwise. }
\end{array}\right.
$$

The corresponding mean progeny representation graph is shown in Figure 6.3.

Proposition 3. For the branching process described in Example 3, 
(a)

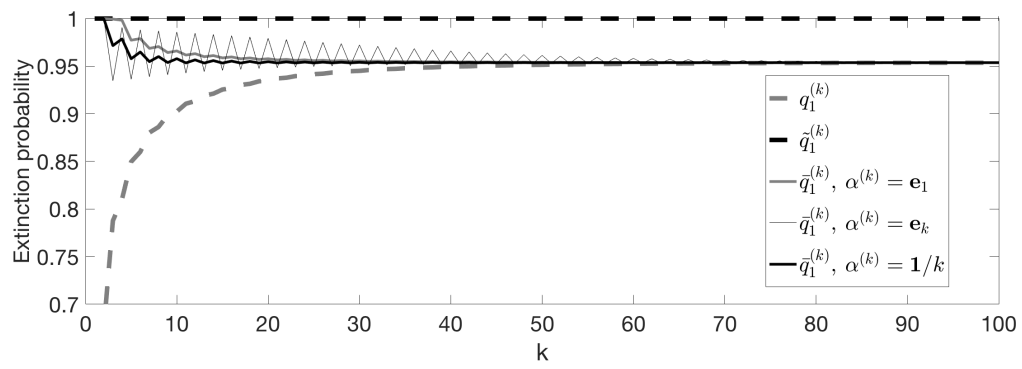

(b)

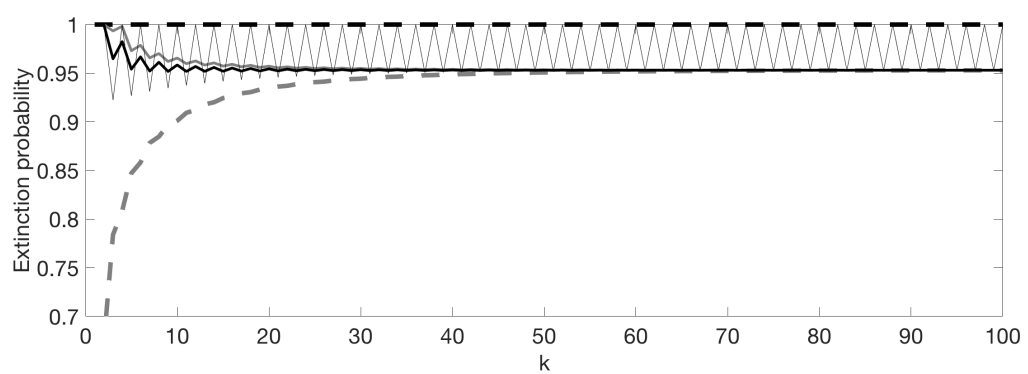

(c)

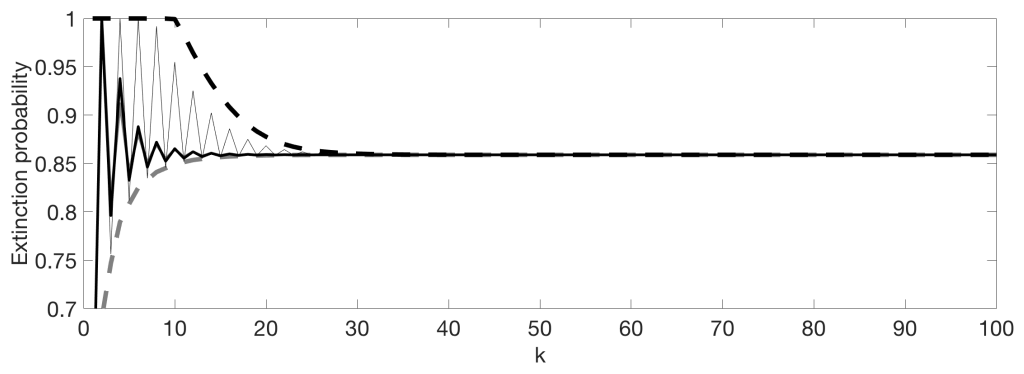

(d)

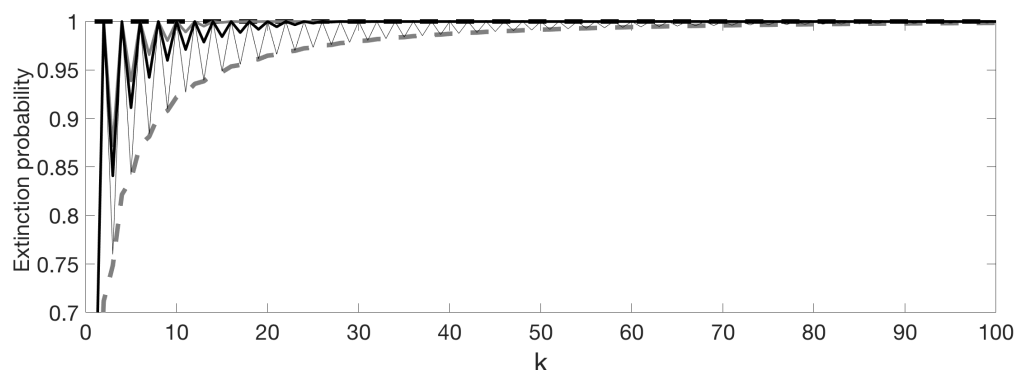

Figure 6.2: Sequences of extinction probabilities $\tilde{q}_{1}^{(k)}, q_{1}^{(k)}$ and $\bar{q}_{1}^{(k)}$ for different replacement distributions and different parameters values, corresponding to Example 2. Details are given in the text. 


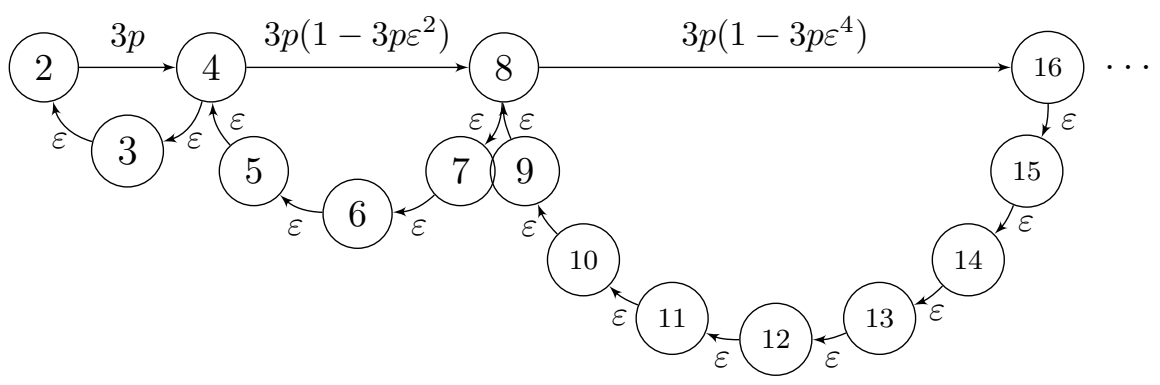

Figure 6.3: The mean progeny representation graph corresponding to Example 3.

(i) $\tilde{\boldsymbol{q}}=\mathbf{1}$,

(ii) if $p>1 / 3$ then $\boldsymbol{q}<\mathbf{1}$, and

(iii) if $p<2 / 3$ and $\boldsymbol{\alpha}^{(k)}=\mathbf{1} / k$, then $\lim _{k \rightarrow \infty} \overline{\boldsymbol{q}}^{(k)}=\mathbf{1}$.

When $p>2 / 3$, one can show that $\bar{m}^{(k)} \rightarrow \infty$; while this implies $\overline{\boldsymbol{q}}^{(k)}<\mathbf{1}$ for all $k$ large enough, it alone does not rule out the case where $\overline{\boldsymbol{q}}^{(k)} \rightarrow \mathbf{1}$. We now give a numerical example to further explore the cases $1 / 3<p<2 / 3$ and $p>2 / 3$.

In Figure 6.4 we plot the first entry of each sequence of extinction probability vectors for the branching processes described in Example 3 with $\varepsilon=1 / 2$ and two different values of $p$. In the upper panel of Figure 6.4 we let $p=1 / 2$. In this case, $1 / 3<p<2 / 3$ and in agreement with Proposition 3 we have $\bar{q}_{1}^{(k)} \rightarrow \tilde{q}_{1} \neq q_{1}$ for $\boldsymbol{\alpha}^{(k)}=\mathbf{1} / k$. In the lower panel we take $p=7 / 9$. In this case, $p>2 / 3$, and Proposition 3 does not provide any information about the convergence of $\bar{q}_{1}^{(k)}$, however, simulations indicate that $\bar{q}_{1}^{(k)} \rightarrow q_{1}<1$.

\section{Appendix A. Computational aspects}

The three sequences of extinction probabilities $\left\{\boldsymbol{q}^{(k)}\right\},\left\{\tilde{\boldsymbol{q}}^{(k)}\right\}$, and $\left\{\overline{\boldsymbol{q}}^{(k)}\right\}$ defined in Section 3 are easy to implement in practice, as we show now. Since the convergences $\boldsymbol{q}^{(k)} \rightarrow \boldsymbol{q}, \tilde{\boldsymbol{q}}^{(k)} \rightarrow \tilde{\boldsymbol{q}}$, and $\overline{\boldsymbol{q}}^{(k)} \rightarrow \boldsymbol{q}$ (under the assumptions of Theorem 1) are pointwise, in order to evaluate the $i$ th entry of the desired extinction probability vector, the chosen sequence of approximating vectors should be computed for $k \geq i$. 



Figure 6.4: Sequences of extinction probabilities $\tilde{q}_{1}^{(k)}, q_{1}^{(k)}$ and $\bar{q}_{1}^{(k)}$ for different replacement distributions and different parameters values, corresponding to Example 3. Details are given in the text. 
For $k \geq 1$, let $\boldsymbol{s}^{[k]}:=\left(s_{1}, \ldots, s_{k}\right)^{\top}$ and $\boldsymbol{G}^{[k]}(\boldsymbol{s}):=\left(G_{1}(\boldsymbol{s}), \ldots, G_{k}(\boldsymbol{s})\right)^{\top}$, and let $\varepsilon$ be some predetermined tolerance error. For any $i \in \mathcal{S}$, the pseudocode for the numerical computation of $q_{i}$ or $\tilde{q}_{i}$ depends on the function $\boldsymbol{u}\left(\boldsymbol{s}^{[k]}\right) \in[0,1]^{\infty}$ as described in (6.2)-(6.4), which determines which of the three sequences is used:

Set $x_{i}^{(\text {old })}:=2, k:=i$

Compute $\boldsymbol{x}^{(k)}$ as the minimal non-negative solution of $\boldsymbol{s}^{[k]}=\boldsymbol{G}^{[k]}\left(\boldsymbol{s}^{[k]}, \boldsymbol{u}\left(\boldsymbol{s}^{[k]}\right)\right)$

While $\left|x_{i}^{(k)}-x_{i}^{(\text {old })}\right|>\varepsilon$ do

$$
k:=k+1
$$

Set $\boldsymbol{x}^{(\text {old })}:=\boldsymbol{x}^{(k)}$

Compute $\boldsymbol{x}^{(k)}$ as the min. non-neg. solution of $\boldsymbol{s}^{[k]}=\boldsymbol{G}^{[k]}\left(\boldsymbol{s}^{[k]}, \boldsymbol{u}\left(\boldsymbol{s}^{[k]}\right)\right)$

\section{endwhile}

Return $x_{i}:=x_{i}^{(k)}$,

where

$$
\begin{array}{r}
\boldsymbol{u}\left(\boldsymbol{s}^{[k]}\right)=\mathbf{0} \rightarrow \boldsymbol{x}^{(k)}=\boldsymbol{q}^{(k)} \text { and } x_{i} \approx q_{i}, \\
\boldsymbol{u}\left(\boldsymbol{s}^{[k]}\right)=\mathbf{1} \rightarrow \boldsymbol{x}^{(k)}=\tilde{\boldsymbol{q}}^{(k)} \text { and } x_{i} \approx \tilde{q}_{i}, \\
\boldsymbol{u}\left(\boldsymbol{s}^{[k]}\right)=\sum_{j=1}^{k} \alpha_{j}^{(k)} s_{j} \mathbf{1} \rightarrow \boldsymbol{x}^{(k)}=\overline{\boldsymbol{q}}^{(k)} \text { and } x_{i} \approx q_{i} .
\end{array}
$$

Note that the linear functional iteration algorithm or the quadratic Newton algorithm can be applied to compute the minimal non-negative solution of the finite system $\boldsymbol{s}^{[k]}=\boldsymbol{G}^{[k]}\left(\boldsymbol{s}^{[k]}, \boldsymbol{u}\left(\boldsymbol{s}^{[k]}\right)\right)$ for each value of $k$.

\section{Appendix B. Proofs of the results related to Examples 2 and 3}

Proof of Lemma 5. We calculate $\bar{m}^{(k)}$ by taking the weighted sum of all first return paths to $k$ in the mean progeny representation graph,

$$
\bar{m}^{(k)}=\bar{M}_{k, k}^{(k)}+\bar{M}_{k, k-1}^{(k)} \bar{M}_{k-1, k}^{(k)}+\bar{M}_{k, k-1}^{(k)} \bar{M}_{k-1, k-2}^{(k)} \bar{M}_{k-2, k-1}^{(k)} \bar{M}_{k-1, k}^{(k)}+\ldots
$$

Observe that the number of these paths, $k \rightarrow(k-1) \rightarrow \cdots \rightarrow(k-1) \rightarrow k$, with length $2(l+1) \leq 2(k-1)$ is given by the Catalan number,

$$
C_{l}=\frac{1}{l+1}\left(\begin{array}{c}
2 l \\
l
\end{array}\right), \quad l \geq 0,
$$


whereas, because no path can fall below type 1 , the number of paths is less than $C_{l}$ when $2(l+1)>2(k-1)$. In these expressions, $(l+1)$ can be interpreted as the total number of negative increments in the paths. Furthermore, the length of each first return path is even, the total number of positive and negative increments of each first return path is equal and each first return path alternates between odd and even states. Hence,

$$
\bar{M}_{k, k}^{(k)}+\sum_{l=0}^{k-2} C_{l}(a c)^{l+1} \leq \bar{m}^{(k)} \leq \bar{M}_{k, k}^{(k)}+\sum_{l=0}^{\infty} C_{l}(a c)^{l+1} .
$$

The infinite series converges when $a c \leq 1 / 4$ and diverges when $a c>1 / 4$. In addition, when $a c \leq 1 / 4$,

$$
\sum_{l=0}^{\infty} \frac{1}{l+1}\left(\begin{array}{c}
2 l \\
l
\end{array}\right)(a c)^{l}=\frac{1}{2}(1-\sqrt{1-4 a c}),
$$

which gives the result.

Proof of Proposition 2. Following an approach analogous to the proof of Proposition 5.1 in [16], we can show that the convergence norm of the mean progeny matrix $M$ is $\nu(M)=2 \sqrt{a c}$. By Proposition 4.1 in [16], we see that $\tilde{\boldsymbol{q}}=\mathbf{1}$ if and only if $a c \leq 1 / 4$.

We first turn our attention to cases (i) and (ii). Observe that the number of first return paths to $k$ of any fixed length is monotone increasing with $k$. This means that the sequences $\left\{\bar{m}^{(2 k+1)}\right\}$ and $\left\{\bar{m}^{(2 k)}\right\}$ are monotonically increasing with respect to $k$. Due to the repetitive structure of the progeny distributions and the relative weakness of type 1 with respect to other odd types, we also have

$$
\bar{q}_{2 k+1}^{(2 k+1)} \geq \bar{q}_{2 k+3}^{(2 k+3)} \quad \text { and } \quad \bar{q}_{2 k}^{(2 k)} \geq \bar{q}_{2 k+2}^{(2 k+2)}
$$

for all $k \geq 1$. If $\lim _{k \rightarrow \infty} \bar{m}^{(2 k+1)}>1$, then there exists $\varepsilon_{1}>0$ and an integer $k_{1}$ such that for all $k \geq k_{1}$,

$$
\bar{q}_{2 k+1}^{(2 k+1)}<1-\varepsilon_{1}
$$

whereas, if $\lim _{k \rightarrow \infty} \bar{m}^{(2 k+1)} \leq 1$ then, for all $k \geq 1$,

$$
\bar{q}_{2 k+1}^{(2 k+1)}=1 .
$$


An equivalent result holds when we take the limit over the even values of $k$.

Next, we have $\inf _{i} q_{i} \geq \inf _{i} p_{i}(\mathbf{0})>0$ which, by Lemma 4 , implies that $\left|\boldsymbol{S}_{k}\right|$ satisfies the dichotomy between extinction and unbounded growth. Therefore, for any arbitrary integer $K \geq 1$,

$$
\limsup _{k \rightarrow \infty}\left(\bar{q}_{i}^{(k)}-q_{i}^{(k)}\right)=c_{i} \limsup _{k \rightarrow \infty} \mathbf{E}_{i}\left(\left(\bar{q}_{k}^{(k)}\right)^{\left|\boldsymbol{S}_{k}\right|}|| \boldsymbol{S}_{k} \mid>K\right)
$$

where $c_{i}=\tilde{q}_{i}-q_{i}=\lim _{k \rightarrow \infty} \mathbf{P}_{i}\left(\left|\boldsymbol{S}_{k}\right|>K\right)$ by Lemmas 2 and 4 , with the same holding when limsup is replaced by liminf. In combination with (6.6) and (6.7) we then obtain

$$
\lim _{k \rightarrow \infty}\left(\bar{q}_{i}^{(2 k+1)}-q_{i}^{(2 k+1)}\right)= \begin{cases}0, & \text { if } \lim _{k \rightarrow \infty} \bar{m}^{(2 k+1)}>1, \\ \tilde{q}_{i}-q_{i}, & \text { if } \lim _{k \rightarrow \infty} \bar{m}^{(2 k+1)} \leq 1\end{cases}
$$

and

$$
\lim _{k \rightarrow \infty}\left(\bar{q}_{i}^{(2 k)}-q_{i}^{(2 k)}\right)= \begin{cases}0, & \text { if } \lim _{k \rightarrow \infty} \bar{m}^{(2 k)}>1, \\ \tilde{q}_{i}-q_{i}, & \text { if } \lim _{k \rightarrow \infty} \bar{m}^{(2 k)} \leq 1\end{cases}
$$

Use of the fact that $\lim _{k \rightarrow \infty} \boldsymbol{q}^{(k)} \rightarrow \boldsymbol{q}$ and Lemma 5 then provides the result.

Consider now case (iii). We apply Proposition 4.5 in [16], which states that if there exists $\lambda \leq 1$ and a row vector $\boldsymbol{x}>\mathbf{0}$ such that $\boldsymbol{x} \mathbf{1}<\infty$ and $\boldsymbol{x} M \leq \lambda \boldsymbol{x}$, then $\boldsymbol{q}=\mathbf{1}$. We let $\boldsymbol{x}=\left(x_{i}\right)_{i \geq 1}$ with $x_{i}=(\sqrt{d})^{(-1)^{i}} x^{1-i}$ for some $x>0$. In this case, $\boldsymbol{x} M \leq \boldsymbol{x}$ is equivalent to $c x^{2}+a \leq x$, that is, $x$ belongs to the interval $[(1-\sqrt{1-4 a c}) / 2 c,(1+\sqrt{1-4 a c}) / 2 c]$. Moreover, $x>1$ ensures $\boldsymbol{x} \mathbf{1}<\infty$. It follows that whenever $1<(1+\sqrt{1-4 a c}) / 2 c$, there exists an $x$ satisfying both conditions, which implies that $\tilde{\boldsymbol{q}}=\boldsymbol{q}=\mathbf{1}$.

Proof of Proposition 3. To prove (i), we consider the type- $2^{k}$ process embedded with respect to $\left\{\tilde{\boldsymbol{Z}}_{n}^{\left(2^{k}\right)}: \varphi_{0}=2^{k}\right\}$, and calculate its mean number of offspring, denoted by $\tilde{m}^{\left(2^{k}\right)}$, for $k \geq 1$. We tackle this by computing the weighted sum of all first return paths to node $2^{k}$ in the mean progeny representation graph illustrated in Figure 6.3 , which we alter by removing all nodes greater than $2^{k}$ to account for the corresponding types being sterile in $\left\{\tilde{\boldsymbol{Z}}_{n}^{\left(2^{k}\right)}\right\}$. Observe that when $k=2,\left(2^{k}=4\right)$ there is a single first return path $4 \rightarrow 3 \rightarrow 2 \rightarrow 4$, thus, $\tilde{m}^{(4)}=3 p \varepsilon^{2}$. When $k>2$, we calculate $\tilde{m}^{\left(2^{k}\right)}$ recursively as follows. First observe that each first return path to $2^{k}$ begins 
(a)

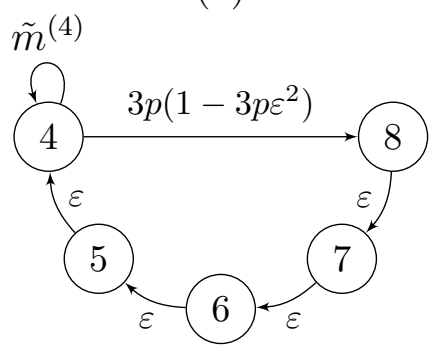

(b)

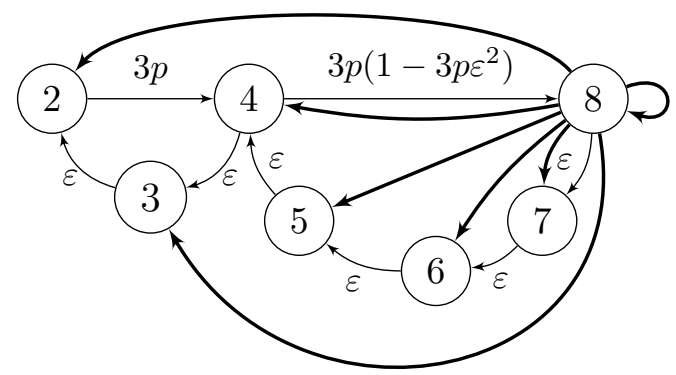

Figure 6.5: A visual representation of the mean progeny matrix in Example 3. Bold edges in (b) have weight $\frac{3 p\left(1-3 p \varepsilon^{4}\right)}{7}$.

with the sequence of edges $2^{k} \rightarrow\left(2^{k}-1\right) \rightarrow \cdots \rightarrow\left(2^{k-1}+1\right) \rightarrow 2^{k-1}$, and ends with the edge $2^{k-1} \rightarrow 2^{k}$. Additionally, the remainder of each path (or the midsection) can be partitioned into the first return paths that were summed to obtain $\tilde{m}^{\left(2^{k-1}\right)}$. That is, for the purpose of calculating $\tilde{m}^{\left(2^{k}\right)}$, in the mean progeny matrix representation graph, nodes of type $<2^{k-1}$ can then be replaced by a loop to type $2^{k-1}$ with weight $\tilde{m}^{\left(2^{k-1}\right)}$. See Figure 6.5 (a) for an illustration when $k=3$. It can then be shown that,

$$
\tilde{m}^{\left(2^{k}\right)}=\varepsilon^{2^{k-1}}\left[1+\tilde{m}^{\left(2^{k-1}\right)}+\left(\tilde{m}^{\left(2^{k-1}\right)}\right)^{2}+\ldots\right] 3 p\left(1-3 p \varepsilon^{2^{k-2}}\right) .
$$

We can then prove by induction that $\tilde{m}^{\left(2^{k}\right)}=\varepsilon^{2^{k-1}} 3 p$, which leads to $\tilde{m}^{\left(2^{k}\right)}<$ 1 for all $k \geq 1$ since, by assumption, $3 p \varepsilon^{\left(2^{k-1}\right)} \leq 3 p \varepsilon^{2}<1$. Combining this with the fact that for all $k \geq 2,\left\{\tilde{\boldsymbol{Z}}_{n}^{\left(2^{k}\right)}\right\}$ is irreducible, we obtain $\tilde{\boldsymbol{q}}^{\left(2^{k}\right)}=\mathbf{1}$ for all $k \geq 1$. Since $\left\{2^{k}\right\}_{k \geq 1}$ is an infinite subsequence of $\mathbb{N}$, the result then follows from the fact that $\lim _{k \rightarrow \infty} \tilde{\boldsymbol{q}}^{(k)}=\tilde{\boldsymbol{q}}$.

We now prove (ii). If $p>1 / 3$, then there exists $\gamma>0$ such that $p=$ $1 / 3+\gamma$, and there exist an integer $N$ and a constant $0<C<3 \gamma$ such that $3 p\left(1-3 p \varepsilon^{i / 2}\right)=(1+3 \gamma)\left(1-3 p \varepsilon^{i / 2}\right)>1+C$ for all $i \geq 2^{N}$ (since $3 p \varepsilon^{i / 2}$ becomes arbitrarily close to 0 as $i$ increases). By disregarding all types $j$ such that $j \leq 2^{N}$ or $j \notin\left\{2^{k}\right\}_{k \geq 2}$ it can be shown that $\left\{\left|\boldsymbol{Z}_{n}\right|: \varphi_{0}=2^{N}\right\}$ is stochastically greater than the Galton-Watson process with progeny generating function $G(s)=(1 / 3+C / 3) s^{3}+(2 / 3-C / 3)$. Since $G^{\prime}(1)>1$, we have $q_{2^{N}}<1$. The result follows from irreducibility. 
To prove (iii) we consider the type- $2^{k}$ process embedded in $\left\{\overline{\boldsymbol{Z}}_{n}^{\left(2^{k}\right)}: \varphi_{0}=\right.$ $\left.2^{k}\right\}$ and calculate its mean number of offspring $\bar{m}^{\left(2^{k}\right)}$. To account for the instantaneous replacement of all individuals of type $>2^{k}$ with a type uniformly distributed on $\left\{2, \ldots, 2^{k}\right\}$, the graph illustrated in Figure 6.3 is altered by removing all nodes greater than $2^{k}$ and adding an edge of weight $3 p\left(1-3 p \varepsilon^{2^{k-1}}\right) /\left(2^{k}-1\right)$ from node $2^{k}$ to all the remaining nodes. See Figure 6.5 (b) for an illustration when $k=3$. We then calculate the weighted sum of all first return paths to $2^{k}$. Note that these paths include those involved in the computation of $\tilde{m}^{\left(2^{k}\right)}$. More specifically, if we let $f_{i, k}$ be the weighted sum of all first passage paths from $2^{i}$ to $2^{k}$, then

$$
\bar{m}^{\left(2^{k}\right)}=\tilde{m}^{\left(2^{k}\right)}+\frac{3 p\left(1-3 p \varepsilon^{2^{k-1}}\right)}{2^{k}-1}\left(1+\sum_{j=1}^{k-1} f_{k-j, k}\left(\sum_{i=0}^{2^{k-j}-1} \varepsilon^{i}\right)\right) .
$$

By applying a recursive argument analogous to the proof of (i), it can then be shown that $f_{i, k}=(3 p)^{k-i}$. Assuming $p \neq 1 / 3$ we have,

$$
\begin{aligned}
\bar{m}^{\left(2^{k}\right)} & =3 p \varepsilon^{2^{k-1}}+\frac{3 p\left(1-3 p \varepsilon^{2^{k-1}}\right)}{2^{k}-1}\left(1+\frac{1}{1-\varepsilon} \sum_{j=1}^{k-1}(3 p)^{j}\left(1-\varepsilon^{2^{k-j}}\right)\right) \\
& \leq 3 p \varepsilon^{2^{k-1}}+\frac{3 p}{2^{k}-1}\left(\frac{1-(3 p)^{k}}{(1-3 p)(1-\varepsilon)}+\varepsilon\right) \rightarrow 0 \quad \text { as } k \rightarrow \infty
\end{aligned}
$$

when $p<2 / 3$. When $p=1 / 3$ it can be shown that an equivalent result holds. This demonstrates that $\lim _{k \rightarrow \infty} \bar{m}^{\left(2^{k}\right)}=0$ when $p<2 / 3$. The proof that $\bar{m}^{(k)} \rightarrow 0$ is an extension of the same method and is omitted. Therefore, there exists an integer $N$ such that for all $k>N, \bar{m}^{(k)}<1$ hence $\overline{\boldsymbol{q}}^{(k)}=\mathbf{1}$, which shows that $\overline{\boldsymbol{q}}^{(k)} \rightarrow \mathbf{1}$ as $k \rightarrow \infty$.

\section{Acknowledgements}

The authors are grateful to the anonymous reviewers for their constructive comments, which helped us to improve the manuscript. The authors would like to acknowledge the support of the Australian Research Council (ARC) through the Centre of Excellence for the Mathematical and Statistical Frontiers (ACEMS). Sophie Hautphenne would further like to thank the ARC for support through Discovery Early Career Researcher Award DE150101044. 


\section{References}

[1] Athreya, K.B. and Ney, P.E. (1972). Branching Processes. Springer, Berlin.

[2] Athreya, K. B. (1993). Rates of decay for the survival probability of a mutant gene II The multitype case. Journal of Mathematical Biology, 32.1: $45-53$.

[3] Kimmel, M. and Axelrod, D.E. (2002). Branching Processes in Biology. Springer, New York.

[4] Bertacchi, D. and ZuccA, F. (2009). Characterization of critical values of branching random walks on weighted graphs through infinitetype branching processes. Journal of Statistical Physics. 134.1: 53-65.

[5] Bertacchi, D. and Zucca, F. (2013). Recent results on branching random walks. Statistical Mechanics and Random Walks: Principles, Processes and Applications, Nova Science Publishers, 289-340.

[6] Bertacchi, D. and ZuccA, F. (2014). Strong local survival of branching random walks is not monotone. Advances in Applied Probability, 46.2: $400-421$.

[7] Bertacchi, D. and ZucCA, F. (2017). A generating function approach to branching random walks. Brazilian Journal of Probability and Statistics, 31.2: 229-253.

[8] Braunsteins, P. and Hautphenne, S. (2017). Extinction in lower Hessenberg branching processes with countably many types. arXiv preprint arXiv:1706.02919.

[9] Comets, F. and Popov, S. (2007). On multidimensional branching random walks in random environment. The Annals of Probability, 68114.

[10] Gantert, N. and Müller, S. (2006). The critical branching Markov chain is transient. Markov Process and Related Fields, 12.4, 805-814.

[11] Gantert, N., Müller, S., Popov, S. and Vachkovskaia, M. (2010). Survival of branching random walks in random environment. Journal of Theoretical Probability, 23.4, 1002-1014. 
[12] Gibson, D. and Seneta E. (1987). Augmented truncations of infinite stochastic matrices. Journal of Applied Probability, 600-608.

[13] Gibson, D. and Seneta E. (1987). Monotone infinite stochastic matrices and their augmented truncations. Stochastic Processes and their Applications, 24.2, 287-292.

[14] Haccou, P., Jagers, P. and Vatutin, V. A. (2005). Branching processes: variation, growth, and extinction of populations. No. 5. Cambridge University Press.

[15] Harris, T. E. (2002). The theory of branching processes. Courier Corporation.

[16] Hautphenne, S., Latouche, G. and Nguyen, G. (2013). Extinction probabilities of branching processes with countably infinitely many types. Advances in Applied Probability, 45.4, 1068-1082.

[17] Heyman, D. P.(1991). Approximating the stationary distribution of an infinite stochastic matrix. Journal of Applied Probability, 96-103.

[18] Jagers, P. (1975). Branching Processes with Biological Applications. Wiley, London.

[19] JAGers, P. (1989). General branching processes as Markov fields. Stochastic Processes and their Applications, 32.2, 183-212.

[20] JAGERs, P. (1992). Stabilities and instabilities in population dynamics. Journal of Applied Probability, 29, 770-780.

[21] Lindo, A, and Sagitov, S. (2016). General linear-fractional branching processes with discrete time. arXiv preprint arXiv:1510.06859v2

[22] Lò̀ve, M. (1955). Probability Theory; Foundations, Random Sequences. New York: D. Van Nostrand Company.

[23] Mode, C.J. (1971). Multitype Branching Processes. Elsevier, New York.

[24] Moy, S.-T. C. (1966). Ergodic properties of expectation matrices of a branching process with countably many types. J. Math. and Mech. 16, 1201-1225. 
[25] Moy, S.-T. C. (1967). Extensions of a limit theorem of Everett, Ulam and Harris on multitype branching processes to a branching process with countably many types. Ann. Math. Statist. 38, 992-999.

[26] Moyal, J. E. (1962). Multiplicative population chains. Proceedings of the Royal Society of London. Series A, Mathematical and Physical Sciences 266, 518-526.

[27] MüLLER, S. (2008). A criterion for transience of multidimensional branching random walk in random environment. Electronic Journal of Probability 13, 1189-1202.

[28] Sagitov, S. (2013). Linear-fractional branching processes with countably many types. Stochastic Processes and their Applications 123.8, 2940-2956.

[29] Seneta, E. (2006) Non-negative matrices and Markov chains. Springer Science and Business Media.

[30] Spataru, A. (1989). Properties of branching processes with denumerably many types. Revue Roumaine de Mathématiques Pures et Appliquées (Romanian Journal of Pure and Applied Mathematics), 34, $747-759$.

[31] Steel, M. (2015). Reflections on the extinction-explosion dichotomy. Theoretical Population Biology, 101, 61-66.

[32] Tetzlaff, G. T. (2005). Criticality in discrete time branching processes with not uniformly bounded types. Rev. Mat. Apl., 24, 25-36.

[33] Vere-Jones, D. (1967). Ergodic properties of nonnegative matrices. I. Pacific Journal of Mathematics, 22.2, 361-386.

[34] Vere-Jones, D. (1968). Ergodic properties of nonnegative matrices. II. Pacific Journal of Mathematics, 26.3, 601-620.

[35] ZuCCA, F. (2011). Survival, extinction and approximation of discretetime branching random walks. Journal of Statistical Physics, 142.4, 726-753. 


\section{University Library}

\section{- M M N E R VA A gateway to Melbourne's research publications}

Minerva Access is the Institutional Repository of The University of Melbourne

Author/s:

Braunsteins, P;Decrouez, G;Hautphenne, S

Title:

A pathwise approach to the extinction of branching processes with countably many types

Date:

2019-03-01

Citation:

Braunsteins, P., Decrouez, G. \& Hautphenne, S. (2019). A pathwise approach to the extinction of branching processes with countably many types. STOCHASTIC PROCESSES AND THEIR APPLICATIONS, 129 (3), pp.713-739. https://doi.org/10.1016/j.spa.2018.03.013.

Persistent Link:

http://hdl.handle.net/11343/249511 